\title{
Co-Granulated and Blended Zinc Fertilizer Comparison for Corn and Soybean
}

\author{
Matthew Caldwell ${ }^{1}$, Kelly A. Nelson ${ }^{2} \&$ Manjula Nathan $^{1}$ \\ ${ }^{1}$ College of Agriculture, Food and Natural Resources, Division of Plant Sciences, University of Missouri, \\ Columbia, Missouri, USA \\ ${ }^{2}$ Greenley Research Center, Division of Plant Sciences, University of Missouri, Novelty, Missouri, USA \\ Correspondence: Kelly A. Nelson, University of Missouri Greenley Research Center, 64399 Greenley Place, \\ Novelty, MO 63460, USA. Tel: 660-739-4410. E-mail: nelsonke@missouri.edu
}

Received: August 30, $2016 \quad$ Accepted: October 10, $2016 \quad$ Online Published: November 15, 2016

doi:10.5539/jas.v8n12p9 URL: http://dx.doi.org/10.5539/jas.v8n12p9

\begin{abstract}
A new co-granulated formulation of monoammonium phosphate (MAP) including $\mathrm{S}$ and $\mathrm{Zn}$ could allow for more uniform nutrient distribution. A six site-year study evaluated the effects of blended phosphorus $(\mathrm{P})$ sources [MAP and diammonium phosphate (DAP)] and zinc amounts $\left(0,2.2\right.$, and $\left.5.6 \mathrm{~kg} \mathrm{Zn} \mathrm{ha}{ }^{-1}\right)$ compared to co-granulated fertilizer, MicroEssentials ${ }^{\circledR}$ Sulfur-10 $\left(\right.$ MES10 $\left.^{\mathrm{TM}}\right)(12-40-0-10 \mathrm{~S})$ and MicroEssentials Sulfur and Zinc $\left(\mathrm{MESZ}^{\mathrm{TM}}\right)(12-40-0-10 \mathrm{~S}-1 \mathrm{Zn})$, on corn and soybean response. Fertilizers were broadcast applied for corn and the carry-over effect on soybean was determined. Ear leaf P, S, and Zn concentrations at Novelty in 2013 and 2014 were within the sufficiency range regardless of treatment, even though initial soil test values were low-medium. Yields were similar to the $\mathrm{N}$ only control for all site-years except at Novelty in 2013, where $\mathrm{MAP}+\mathrm{ZnSO}_{4}$ at $2.2 \mathrm{~kg} \mathrm{Zn} \mathrm{ha}^{-1}, \mathrm{MAP}+\mathrm{Super} \mathrm{Zn}$ at $5.5 \mathrm{~kg} \mathrm{Zn} \mathrm{ha}^{-1}$, and DAP+AMS were 540 to $570 \mathrm{~kg} / \mathrm{ha}$ greater. The amount of $\mathrm{Zn}$ fertilizer ( $\left.2.2 \mathrm{vs} .5 .6 \mathrm{~kg} \mathrm{Zn} \mathrm{ha}^{-1}\right)$ also showed no significant effect on yield. Applications of $\mathrm{P}$ or $\mathrm{Zn}$ generally increased their concentrations in post-harvest soil samples. Fertilizer applied for corn indicated some differences in soybean plant nutrient concentrations, but it had no effect on total plant nutrient uptake, grain yield or quality. At Novelty, soybean plant $\mathrm{Zn}$ concentration was greater at $5.6 \mathrm{~kg} \mathrm{Zn} \mathrm{ha}{ }^{-1}$ compared to 2.2 $\mathrm{kg} \mathrm{Zn} \mathrm{ha}{ }^{-1}$, while Albany showed an increase in whole soybean plant $\mathrm{Zn}$ concentration with SuperZn compared to $\mathrm{ZnSO}_{4}$. Carry-over fertilizer from corn showed limited effects on soybean response the following year.
\end{abstract}

Keywords: corn, fertilizer, phosphorus, soybean, sulfur, and zinc

\section{Introduction}

Zinc ( $\mathrm{Zn}$ ) is essential to plant survival, with the average plant containing $20 \mathrm{ppm}$ of the micronutrient based on dry weight (Mahler, 2004). Typical soils can contain 0.3 to $2.0 \mathrm{ppm}$ (Mahler, 2004) of plant-available Zn, which is the most common deficient micronutrient in high pH soils (Graham, Asher, \& Hynes, 1992). Zn is found in N metabolism pathways that can affect protein synthesis (Fageria, 2004). Deficiencies can cause interveinal chlorosis, bronzing, internode shortening, and epinasty. In severe deficiencies, the root apex can become necrotic Although $\mathrm{Zn}$ is mobile in the plant, its mobility is poor and deficiency symptoms appear first in the upper, young plant leaves. Since $\mathrm{Zn}$ is a micronutrient, $\mathrm{Zn}$ toxicity is possible, but unlikely (Broadley, White, Hammond, Zelko, \& Lux, 2007). Zn fertilizers are available in three major forms: $\mathrm{Zn}$ chelate, $\mathrm{ZnO}$, and $\mathrm{ZnSO}_{4}$ (Schulte, 2004). Water solubility greatly influences the availability and effectiveness of $\mathrm{Zn}$ fertilizer. In $\mathrm{Zn}$ chelate, commonly sold as ZnEDTA, a large organic molecule surrounds Zn and keeps it from leaching, oxidizing, and precipitating (Schulte, 2004). Zinc sulfate is the most common form, due to its low cost and greater solubility (Schulte, 2004), and has traditionally been a steadfast source in Zn fertilizer (Olsen, 1982).

Although soil may contain enough $\mathrm{Zn}$ to support a crop through the season, $90 \%$ of the $\mathrm{Zn}$ is in forms that make it unavailable (fixed, insoluble, or unexchangeable) (Broadley et al., 2007). In most soils, only 0.1 to $2 \mu \mathrm{g}$ of $\mathrm{Zn}$ per gram are exchangeable (Broadley et al., 2007). Soils with large phosphate levels can cause an imbalance in a crops' physiology including a reduction in $\mathrm{Zn}$ uptake (Olsen, 1982). This phenomenon is known as P-induced $\mathrm{Zn}$ deficiency (Singh, Karamanos, \& Stewart, 1986). Zinc-phosphorous interactions are well documented (Halim, Wassom, \& Ellis, 1968; Keefer, Singh, Horvath, \& Henderlong, 1972; Rehm, Sorensen, \& Wiese, 1981, 1983; Robson \& Pitman, 1983; Singh, Karamanos, \& Stewart, 1988). Phosphorus fertilizer applied in large amounts 
can induce zinc deficiency in soils with low plant-available zinc (Robson \& Pitman, 1983). In soil, P can decrease zinc's solubility (Huang, Barker, Langridge, Smith, \& Graham, 2000). When P requirements are met in the plant, root growth is reduced and mycorrhizae infection less common (Amijee, Stribley, \& Tinker, 1990). Deficiencies in plants could also be induced by a small concentration of $\mathrm{Zn}$ due to rapid growth response to $\mathrm{P}$. Alternatively, large P-to- $\mathrm{Zn}$ ratios could cause a metabolic imbalance in cells and lead to P-induced $\mathrm{Zn}$ deficiencies (Singh et al., 1988). Zinc deficiency may increase in response to an expression of high-affinity phosphate transporters when $\mathrm{P}$ is deficient, likely because the plant utilizes resources from $\mathrm{Zn}$ for phosphate transporters (Huang et al., 2000). Increasing applications of both $\mathrm{Zn}$ fertilizer and $\mathrm{P}$ could help optimize yield (Schnappinger, Martens, \& Hawkins, 1969), even though crops take up little Zn during the growing season.

More than $30 \%$ of the world's arable land has P-limiting yield potential (Vance, Uhde-Stone, \& Allen, 2003). Phosphorus is an essential plant macronutrient that accounts for 3 to $5 \mathrm{~g} \mathrm{~kg}^{-1}$ of a plant's dry weight (Schalchtman, Reid, \& Ayling, 1998). Phosphorus, a structural component in nucleic acids (DNA, RNA), transfers energy as adenosine triphosphate (ATP) and maintains cell structure with phospholipids. Though abundant in soil, $\mathrm{P}$ occurs primarily in a fixed form or outside of the rhizosphere and so is unavailable for plant uptake. When $\mathrm{P}$ is not available in adequate amounts, at least $0.2 \mathrm{mg} \mathrm{L}^{-1}$ in soil solution (Pierzynski, McDowell, Sims, \& Sharpley, 2005), plants can become deficient. Visual signs of P deficiency include overall stunting of the plant, a purple tint from anthocyanin accumulation, and small necrotic leaf spots. Deficiency typically appears in the lower more mature leaves because P is mobile and translocates to new developing tissue (Briskin, Bloom, Taiz, \& Zeiger, 2010). To overcome limited $\mathrm{P}$ availability in the soil and maintain soil test $\mathrm{P}$ levels, todays growers use $\mathrm{P}$ fertilizers commonly available as monoammonium phosphate (MAP) and diammmonium phosphate (DAP).

It is important to optimize sulfur (S) in crops to achieve high yields and grain quality (Tabatabai, 1984). As current environmental laws reduce sulfur emissions from power plants, crop sulfur deficiencies may become more common (Camberato, Maloney, Casteel, \& Johnson, 2012). Correspondingly, the need to apply S fertilizers likely will increase in coming years. Sandy soils with small amounts of organic matter and no-till or heavy residue can increase the likelihood of sulfur deficiencies (Camberato \& Casteel, 2010). Although $\mathrm{S}$ is considered a secondary plant nutrient (primarily because of amount needed), deficiency seriously affects plant growth and yields (Sawyer \& Barker, 2012). As organic matter decomposes, it releases sulfate $\left(\mathrm{SO}_{4}{ }^{-2}\right)$ into the soil through the process of mineralization (Hergert, 2000). For every one $\mathrm{g} \mathrm{kg}^{-1}$ of organic matter, 2.25 to $3.36 \mathrm{~kg} \mathrm{ha}^{-1}$ of sulfate are released annually into the soil, while $10 \mathrm{Mg} \mathrm{ha}^{-1}$ of corn (Zea mays L.) removes approximately 6.11 $\mathrm{kg} \mathrm{ha}^{-1}$ of sulfur in grain alone (Schulte \& Kelling, 1992).

A patented technology employed in co-granulated fertilizers combine nitrogen, $\mathrm{P}, \mathrm{S}$, and $\mathrm{Zn}$ into a single prill (MicroEssentials, Mosaic, Plymouth, MN). This allows for uniform distribution and possibly increased uptake of nutrients across a range of crops. MES10 (MicroEssentials Sulfur) contains MAP plus equal amounts of AMS (ammonium sulfate) and elemental sulfur (S) as $100 \mathrm{~g} \mathrm{~kg}^{-1}$ in the co-granulated material. The sulfate is immediately available for plant uptake, though the elemental sulfur must be oxidized by soil bacteria, which allows for season-long sulfur availability (Schulte \& Kelling, 1992). MESZ is the same formulation as MES plus one percent $\mathrm{ZnO}$. Microessential Sulfur and Zinc (MESZ) utilizes $\mathrm{ZnO}$ as the primary $\mathrm{Zn}$ source (Mosaic, 2007). Zinc oxide has the greatest percent of $\mathrm{Zn}$ at $72-80 \%$ compared to other $\mathrm{Zn}$ sources, but it is less water soluble than $\mathrm{ZnSO}_{4}$.

Researchers have studied the effects of co-granulated fertilizers in Iowa with corn (Sawyer \& Barker, 2009), and in Arkansas with rice (Oryza sativa L.) (Slaton et al., 2010), winter wheat (Triticum aestivum L.) (Freeman, Ruffo, \& Mann, 2014), and canola (Brassica napus L.) (Woolfork, Olson, Mann, \& Perez, 2014); however, results have been mixed. Some studies show limited yield differences (Sawyer \& Barker, 2009), while others indicate an advantage of MES and MESZ compared to a blend of the same nutrients (Slaton et al., 2010). MESZ increased yields $5.7 \%$ compared to MAP and 3.4\% compared to MAP+AMS $+\mathrm{ZnSO}_{4}$ (Freeman et al., 2014). In canola, Woolfolk et al. (2014) reported yield increases of $4 \%$ at $19 \mathrm{~kg} \mathrm{P}_{2} \mathrm{O}_{5} \mathrm{ha}^{-1}$ and $7.1 \%$ at $56 \mathrm{~kg} \mathrm{P}_{2} \mathrm{O}_{5} \mathrm{ha}^{-1}$, which were related to less injury to germinating seedlings. Few studies report on the effects of the new co-granulated fertilizers on corn response in the Midwestern U.S., as well as their carry-over impact on soybean [Glycine $\max$ (L.) Merr.]. The objective of this research was to evaluate corn response to MES10 and MESZ formulations to equivalent blends of DAP or MAP, S, and $\mathrm{Zn}$ at two amounts of $\mathrm{Zn}\left(2.2\right.$ and $\left.5.6 \mathrm{~kg} \mathrm{Zn} \mathrm{ha}^{-1}\right)$ for the impact on corn (ear leaf nutrient concentration at VT, grain yield, grain quality, and changes to soil test nutrient levels post-harvest) and soybean response (population, plant nutrient uptake, yield, and grain quality). 
Table 1. Initial soil characteristics (average \pm 1 standard deviation of the mean) 0-15 cm deep at Albany (2013-2014) and Novelty (2011-2014)

\begin{tabular}{|c|c|c|c|c|c|c|}
\hline \multirow{2}{*}{ Soil characteristics } & \multirow{2}{*}{2011 Novelty } & \multirow{2}{*}{2012 Novelty } & \multicolumn{2}{|c|}{2013} & \multicolumn{2}{|c|}{2014} \\
\hline & & & Novelty & Albany & Novelty & Albany \\
\hline $\mathrm{pH}\left(0.01 \mathrm{M} \mathrm{CaCl}_{2}\right)$ & $6.0 \pm 0.1$ & $6.2 \pm 0.2$ & $5.1 \pm 0.6$ & $5.1 \pm 0.2$ & $5.7 \pm 0.2$ & $5.9 \pm 0.3$ \\
\hline Neutralizable acidity $\left(\mathrm{cmol}_{\mathrm{c}} \mathrm{kg}^{-1}\right)$ & $1.9 \pm 0.2$ & $1.1 \pm 0.4$ & $5.4 \pm 5.5$ & $4.5 \pm 1.1$ & $2.5 \pm 0.1$ & $2.0 \pm 0.9$ \\
\hline Organic matter $\left(\mathrm{g} \mathrm{kg}^{-1}\right)$ & $23 \pm 1$ & $29 \pm 2$ & $20 \pm 2$ & $26 \pm 3$ & $22 \pm 4$ & $29 \pm 1$ \\
\hline Bray 1P $\left(\mathrm{kg} \mathrm{ha}^{-1}\right)$ & $15.7 \pm 2.4(\mathrm{VL})^{\dagger}$ & $15.7 \pm 2.1(\mathrm{VL})$ & $21.9 \pm 8.9(\mathrm{~L})$ & $24.6 \pm 7.5(\mathrm{~L})$ & $25.7 \pm 2.5(\mathrm{~L})$ & $35.3 \pm 4.4(\mathrm{M})$ \\
\hline \multicolumn{7}{|l|}{ Exchangeable (1 $\left.\mathrm{M} \mathrm{NH}_{4} \mathrm{OA}_{\mathrm{C}}\right)$} \\
\hline $\mathrm{Ca}\left(\mathrm{kg} \mathrm{ha}^{-1}\right)$ & $4547 \pm 235$ & $4805 \pm 314$ & $3674 \pm 381$ & $3618 \pm 426$ & $4797 \pm 415$ & $5728 \pm 235$ \\
\hline $\operatorname{Mg}\left(\mathrm{kg} \mathrm{ha}^{-1}\right)$ & $392 \pm 37$ & $347 \pm 34$ & $328 \pm 49$ & $459 \pm 64$ & $412 \pm 52$ & $716 \pm 48$ \\
\hline $\mathrm{K}\left(\mathrm{kg} \mathrm{ha}^{-1}\right)$ & $161 \pm 11$ & $160 \pm 20$ & $128 \pm 38$ & $234 \pm 44$ & $228 \pm 30$ & $206 \pm 16$ \\
\hline $\mathrm{SO}_{4}-\mathrm{S}\left(\mathrm{mg} \mathrm{kg}^{-1}\right)$ & $5.8 \pm 1.1(\mathrm{M})$ & $6.4 \pm 0.7(\mathrm{M})$ & $1.6 \pm 0.3(\mathrm{M})$ & $5.7 \pm 0.4(\mathrm{M})$ & $4.5 \pm 0.2(\mathrm{M})$ & $5.6 \pm 0.6(\mathrm{M})$ \\
\hline $\mathrm{Zn}\left(\mathrm{mg} \mathrm{kg}^{-1}\right)$ & $0.2 \pm 0.1(\mathrm{~L})$ & $0.5 \pm 0.1(\mathrm{~L})$ & $0.3 \pm 0.1(\mathrm{~L})$ & $1.0 \pm 0.3(\mathrm{M})$ & $0.8 \pm 0.6(\mathrm{M})$ & $1.0 \pm 0.6(\mathrm{M})$ \\
\hline $\operatorname{Mn}\left(\mathrm{mg} \mathrm{kg}^{-1}\right)$ & $16.7 \pm 0.8$ & $49.3 \pm 7.4$ & $17.2 \pm 1.7$ & - & $19.3 \pm 3.2$ & $9.8 \pm 1.7$ \\
\hline $\mathrm{Fe}\left(\mathrm{mg} \mathrm{kg}^{-1}\right)$ & $38 \pm 1.0$ & $49.3 \pm 7.4$ & $48.3 \pm 12.4$ & - & $40.3 \pm 3.9$ & $43.3 \pm 11.8$ \\
\hline $\mathrm{Cu}\left(\mathrm{mg} \mathrm{kg}^{-1}\right)$ & $0.6 \pm 0.1$ & $0.6 \pm 0.1$ & $0.4 \pm 0.1$ & - & $0.6 \pm 0.1$ & $0.8 \pm 0.1$ \\
\hline $\mathrm{CEC}\left(\mathrm{cmol}_{\mathrm{c}} \mathrm{kg}^{-1}\right)$ & $13.7 \pm 0.8$ & $13.3 \pm 0.7$ & $14.2 \pm 3.2$ & $14.6 \pm 1.1$ & $14.7 \pm 1.2$ & $17.7 \pm 0.7$ \\
\hline
\end{tabular}

Note. ${ }^{\dagger}$ Abbreviations: L, low; M, medium; VL, very low (Buchholz et al., 2004).

\section{Materials and Methods}

\subsection{Corn}

In 2011 and 2012, field research was conducted at the Greenley Memorial Research Center $\left(39^{\circ} 56^{\prime} \mathrm{N}, 9^{\circ} 3^{\prime} \mathrm{W}\right)$ near Novelty, Missouri. In 2013 and 2014, field research was also conducted at the Hundley-Whaley Center $\left(40^{\circ} 14^{\prime} \mathrm{N}, 94^{\circ} 20^{\prime} \mathrm{W}\right)$ near Albany, Missouri. Soil test Bray $1 \mathrm{P}$ at Novelty and Albany was very low $\left(15 \mathrm{~kg} \mathrm{ha}^{-1}\right)$ to medium (36 kg ha ${ }^{-1}$ ) (Table 1). The Novelty sites were a Putnam silt loam (fine, smectitic, mesic Vertic Albaqualfs), while Albany sites were a Grundy silt loam (fine, smectitic, mesic Aquertic Argiudolls). Treatments were arranged in a randomized complete block design with five replications at Novelty and four replications at Albany. Initial $(15 \mathrm{~cm})$ soil samples from each replication were collected and analyzed by the University of Missouri Soil and Plant Testing Laboratory using the recommended soil test procedures for Missouri (Nathan, Stecker, \& Sun, 2012).

Fertilizer treatments were applied pre-plant for corn in the corn-soybean rotation. Treatments included $\mathrm{P}$ source (MAP or DAP), $\mathrm{Zn}$ rate (2.2 and $5.6 \mathrm{~kg} \mathrm{Zn} \mathrm{ha}^{-1}$ ), and multiple fertilizer technologies (traditional blends or co-granulated fertilizers). Zinc rates were in line with other research showing corn yield increases (Schnappinger et al., 1969). In 2013 and 2014, SuperZn (liquid Zn oxide) (1-0-0-0-40Zn) (Helena, Collierville, TN) was impregnated on the dry fertilizer prills and added at both Novelty and Albany. Co-granulated fertilizers included MicroEssentials Sulfur and Zn (MESZ) (12-40-0-10S-1Zn) and MicroEssentials Sulfur (MES10) (12-40-0-10S-1Zn). In 2013 and 2014, Novelty and Albany had sixteen treatments, including: non-treated control (no fertilizer), nitrogen (N) only, DAP, DAP+ ammonium sulfate (AMS), DAP+ZnSO at $_{2} .2 \mathrm{~kg} \mathrm{Zn} \mathrm{ha}^{-1}$, $\mathrm{DAP}+\mathrm{ZnSO}_{4}$ at $5.6 \mathrm{~kg} \mathrm{Zn} \mathrm{ha}^{-1}$, DAP+SuperZn at $2.2 \mathrm{~kg} \mathrm{Zn} \mathrm{ha}^{-1}$, DAP+SuperZn at $5.6 \mathrm{~kg} \mathrm{Zn} \mathrm{ha-1,} \mathrm{MAP,}$ $\mathrm{MAP}+\mathrm{AMS}, \mathrm{MAP}+\mathrm{ZnSO}_{4}$ at $2.2 \mathrm{~kg} \mathrm{Zn} \mathrm{ha}^{-1}, \mathrm{MAP}^{2} \mathrm{ZnSO}_{4}$ at $5.6 \mathrm{~kg} \mathrm{Zn} \mathrm{ha}{ }^{-1}, \mathrm{MAP}+\operatorname{SuperZn}$ at $2.2 \mathrm{~kg} \mathrm{Zn} \mathrm{ha}^{-1}$, MAP+SuperZn at $5.6 \mathrm{~kg} \mathrm{Zn} \mathrm{ha-1,} \mathrm{MES10,} \mathrm{and} \mathrm{MESZ.} \mathrm{In} 2011$ and 2012 at Novelty, SuperZn or DAP+AMS were not included, resulting in a total of eleven treatments.

Corn was planted in April or May, depending on yearly weather conditions using no-till (Novelty) or minimum tillage (Albany) into 3 by 9 to $15 \mathrm{~m}$ plots, with $76 \mathrm{~cm}$ row spacing. Corn followed soybean at all sites, except in 2013 at Albany, a continuous corn site. Management information is available in Table 2.

In 2013 and 2014 at Novelty, ten ear leaves were randomly selected from the middle two rows of each plot. Ear

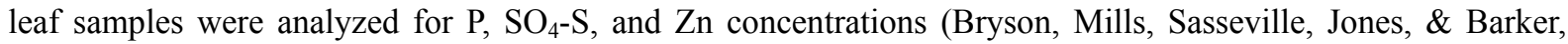
2014). The two middle rows of the four-row corn plots were harvested using a plot combine (Wintersteiger Delta, Salt Lake City, UT or Massey 8, Haven, KS) measuring corn grain yields and moisture content. Corn grain yields 
were adjusted to $150 \mathrm{~g} \mathrm{~kg}^{-1}$ prior to analysis. Individual plot grain samples were collected during harvest and evaluated for oil, protein, and starch using a near infrared (NIR) spectroscopy (Foss Infratec, Eden Prairie, MN).

\subsection{Soybean}

Soybean was planted into the same plots as corn in April or May, depending on yearly weather conditions. The fields were no-till (Novelty) or minimum tillage (Albany), and plots were 3 by 9 to $15 \mathrm{~m}$. In 2015, soybean was planted in July due to an extremely wet spring. Soybean was planted in $76 \mathrm{~cm}$ wide rows at Albany and in $19 \mathrm{~cm}$ rows at Novelty. Soybean followed corn in all years. Management information is available in Table 3. Whole plant tissue samples were taken in 2014 and 2015 at Novelty and Albany at R6 (Fehr \& Caviness, 1971). Quadrats $\left(0.23 \mathrm{~m}^{2}\right)$ were randomly selected from the middle two rows of each plot. The plant samples were ground, dried, and analyzed with standard extraction methods for P, S, and Zn concentrations (Bryson et al., 2014).

To determine soybean grain yields and moisture content, the two middle rows of soybean were harvested at Albany (Massey 8, Haven, KS), and a $1.5 \mathrm{~m}$ wide section of the plot (four, $38 \mathrm{~cm}$ wide rows) was harvested at Novelty (Wintersteiger Delta, Salt Lake City, UT). During harvest, individual plot grain samples were collected and evaluated for oil and protein concentration using near infrared (NIR) spectroscopy (Foss Infratec, Eden Prairie, MN). Soybean yields were adjusted to $130 \mathrm{~g} \mathrm{~kg}^{-1}$ prior to analysis.

\subsection{Statistical Protocol}

All corn data were analyzed with the Statistical Analysis System (SAS Institute, Cary, NC) using PROC GLIMMIX, and means were separated using Fisher's Protected LSD $(P=0.05)$. Corn data for Novelty in 2011 and 2012 were analyzed separately from 2013 and 2014 data due to the addition of SuperZn in the latter years. Data were combined by year and location when appropriate. Soybean data were combined over years for individual sites for all measurements. Planned contrasts were used to compare $\mathrm{Zn}$ sources (SuperZn vs. $\mathrm{ZnSO}_{4}$ ) and $\mathrm{Zn}$ amounts (2.2 vs. $\left.5.6 \mathrm{~kg} \mathrm{Zn} \mathrm{ha}{ }^{-1}\right)$.

\section{Results and Discussion}

\subsection{Growing Conditions}

Research at Novelty from 2011 to 2014 and at Albany in 2013 and 2014 experienced a wide range of precipitation during the growing seasons (March 31 to September 29) (Figure 1). At Novelty in 2011, corn experienced an abnormally dry spring (USDM, 2015) followed by average summer precipitation (532 mm) throughout the growing season. In 2012, the Midwestern U.S. experienced an extreme drought (USDM, 2015), with Novelty receiving only $273 \mathrm{~mm}$ of precipitation during the growing season. In 2013, precipitation was average at Novelty $(453 \mathrm{~mm})$, and above average at Albany (607 $\mathrm{mm})$. 

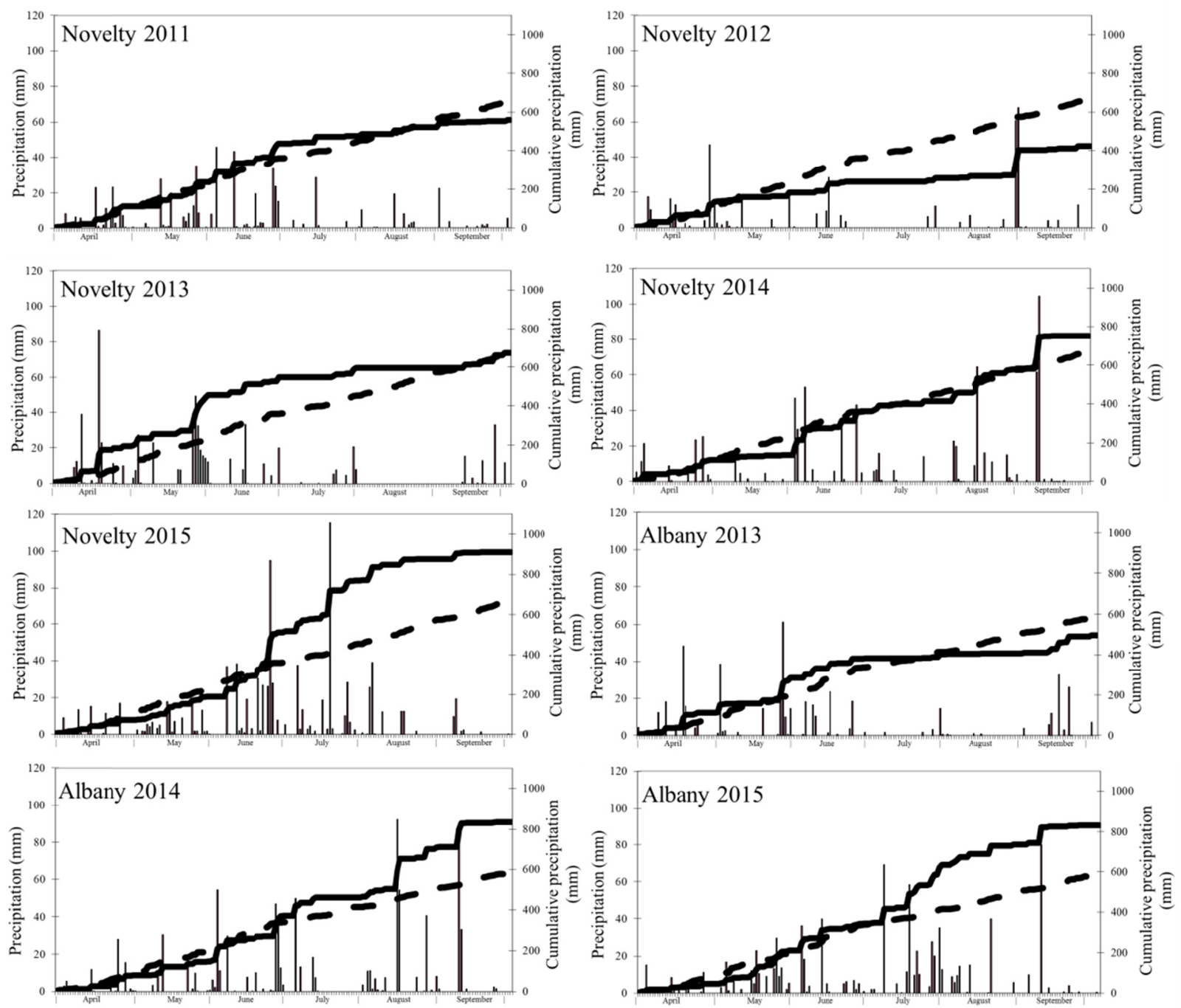

Figure 1. Precipitation over the six site-years for corn (2011-2014) and the following soybean (2012-2015) crop.

Bars represent individual precipitation events (left vertical axis). The solid line represents cumulative precipitation throughout the season, and the dashed line represents 10-year average precipitation

(right vertical axis) 
Table 2. Field and management information for corn sites at Novelty (2011-2014) and Albany (2013-2014)

\begin{tabular}{|c|c|c|c|c|c|c|}
\hline \multirow{2}{*}{ Management information } & \multirow{2}{*}{2011 Novelty } & \multirow{2}{*}{2012 Novelty } & \multicolumn{2}{|c|}{2013} & \multicolumn{2}{|c|}{2014} \\
\hline & & & Novelty & Albany & Novelty & Albany \\
\hline Plot size (m) & 3 by 12 & 3 by 12 & 3 by 15 & 3 by 11 & 3 by 15 & 3 by 11 \\
\hline Hybrid or cultivar & DKC 63-84 & DKC 63-84 & DKC 63-25 VT3 & DKC 64-69 & DKC $63-25$ & DK 64-69 \\
\hline Planting date & 12 Apr. & 2 Apr. & 15 May & 14 May & 18 Apr. & 5 May \\
\hline Seeding rate (seeds ha ${ }^{-1}$ ) & 76,600 & 79,100 & 81,1500 & 71,700 & 81,500 & 74,100 \\
\hline Harvest date & 22 Sep. & 28 Aug. & 7 Oct. & 10 Oct. & 10 Oct. & 16 Oct. \\
\hline Maintenance fertilizer & 31 Mar. 2011 & 18 Nov. 2011 & & & 11 Nov. 2013 & NA \\
\hline Nitrogen & $200 \mathrm{~kg} \mathrm{~N} \mathrm{ha}^{-1}(\mathrm{AA})^{\dagger}$ & $\begin{array}{l}213 \mathrm{~kg} \mathrm{~N} \mathrm{ha}^{-1}(\mathrm{AA})+ \\
\text { nitrapyrin at } 2.34 \mathrm{~L} \mathrm{ha}^{-1}\end{array}$ & $200 \mathrm{~kg} \mathrm{~N} \mathrm{ha}^{-1}$ (AA) & $\begin{array}{l}200 \mathrm{~kg} \mathrm{~N} \mathrm{ha}^{-1} \\
(\mathrm{AN})\end{array}$ & $245 \mathrm{~kg} \mathrm{~N} \mathrm{ha}^{-1}$ & $\begin{array}{l}200 \mathrm{~kg} \mathrm{~N} \mathrm{ha}^{-1} \\
(\mathrm{AN})\end{array}$ \\
\hline P-S-Zn application & 6 May & 28 Nov. 2011 & 29 Apr. & 7 May & 25 Mar. & 5 May \\
\hline $\begin{array}{l}\text { Tillage } \\
\text { Weed management }\end{array}$ & No-till & No-till & No-till & Minimum & No-till & Minimum \\
\hline $\begin{array}{l}\text { Burndown/ } \\
\text { Pre-emergence }{ }^{\ddagger}\end{array}$ & $\begin{array}{l}5 \text { Apr., glyphosate } \\
1.2 \mathrm{~kg} \text { ae ha }{ }^{-1}+ \\
\text { saflufenacil } 0.03 \mathrm{~kg} \\
\text { ai ha }{ }^{-1}+ \\
\text { dimethenameid-P } \\
0.2 \mathrm{~kg} \text { ai. } \mathrm{ha}^{-1}+ \\
\text { AMS } 18 \mathrm{~g} \mathrm{~L}^{-1}\end{array}$ & $\begin{array}{l}19 \text { Mar., saflufenacil } \\
0.03 \text { kg ai. ha }{ }^{-1}+ \\
\text { dimethenameid-P } 0.2 \mathrm{~kg} \\
\text { ai ha }{ }^{-1}+\text { glyphosate } 1.2 \\
\mathrm{~kg} \text { ae ha }{ }^{-1}+\text { AMS } 18 \mathrm{~g} \\
\mathrm{~L}^{-1}\end{array}$ & $\begin{array}{l}17 \text { May, atrazine } 1.5 \\
\mathrm{~kg} \text { ai ha }{ }^{-1}+ \\
\text { S-metolachlor } 1.5 \mathrm{~kg} \\
\text { ai ha }{ }^{-1}+\text { mesotrione } \\
0.2 \mathrm{~kg} \mathrm{ai} \mathrm{ha}^{-1}+\text { MSO } \\
1 \% \mathrm{v} / \mathrm{v}+\text { UAN } 2.34 \\
\mathrm{~L} \mathrm{ha}^{-1}+\text { Glyphosate } \\
1.2 \mathrm{~kg}_{\text {a.e. }} \mathrm{ha}^{-1}\end{array}$ & $\begin{array}{l}14 \text { May, atrazine } \\
1.5 \mathrm{~kg} \text { ai ha } \\
\end{array}$ & $\begin{array}{l}13 \text { Nov. } 2013 \text {, } \\
\text { simazine } 1.1 \mathrm{~kg} \text { ai } \\
\text { ha-1 }+ \text { glyphosate } 0.6 \\
\mathrm{~kg} \text { a.e. } \mathrm{ha}^{-1}+2,4-\mathrm{D} \\
2.7 \mathrm{~kg} \mathrm{ae} \mathrm{ha}+ \\
\text { COC } 2.34 \mathrm{~L} \mathrm{ha}^{-1}\end{array}$ & $\begin{array}{l}5 \text { May, atrazine } \\
1.5 \mathrm{~kg}^{-1} \mathrm{ha}^{-1}+ \\
\mathrm{s}-\mathrm{metolachlor} \\
1.5 \mathrm{~kg}^{-1} \text { ha }^{-1}+ \\
\text { mesotrione } 0.2 \\
\mathrm{~kg} \mathrm{ai} \mathrm{ha}^{-1}+ \\
\text { glyphosate } 1.2 \\
\mathrm{~kg} \text { a.e. ha }\end{array}$ \\
\hline Postemergence & $\begin{array}{l}17 \text { May, acetochlor } \\
3.2 \mathrm{~kg} \text { ai ha }\end{array}$ & $\begin{array}{l}10 \text { May, atrazine } 1.5 \mathrm{~kg} \\
\text { ai ha }{ }^{-1}+\text { S-metolachlor } \\
1.5 \mathrm{~kg}^{-1} \mathrm{ha}^{-1}+ \\
\text { glyphosate } 1.2 \mathrm{~kg} \text { a.e. } \\
\mathrm{ha}^{-1}+0.25 \% \mathrm{v} / \mathrm{v} \mathrm{NIS}\end{array}$ & & $\begin{array}{l}11 \text { June, } \\
\text { glyphosate } 1.2 \\
\text { kg a.e. ha }^{-1}\end{array}$ & $\begin{array}{l}24 \text { May, atrazine } 1.5 \\
\mathrm{~kg} \text { ai ha }{ }^{-1}+ \\
\text { S-metolachlor } 1.5 \mathrm{~kg} \\
\text { ai ha }{ }^{-1}+\text { glyphoshate } \\
1.2 \mathrm{~kg} . \mathrm{e} . \mathrm{ha}^{-1}+ \\
0.25 \% \mathrm{v} / \mathrm{v} \mathrm{NIS}\end{array}$ & \\
\hline Insect management & $\begin{array}{l}17 \text { May, } \\
\text { Lambda-cyhalothrin } \\
0.02 \mathrm{~kg} \text { ai ha }^{-1}\end{array}$ & $\begin{array}{l}10 \text { May, } \\
\text { Lambda-cyhalothrin } \\
0.02 \mathrm{~kg} \mathrm{ha}^{-1}\end{array}$ & NA & NA & NA & NA \\
\hline Disease management & NA & NA & NA & NA & $\begin{array}{l}10 \text { July, } \\
\text { azoxystrobin } 0.08 \mathrm{~kg} \\
\text { ai ha }{ }^{-1}\end{array}$ & NA \\
\hline
\end{tabular}

Note. ${ }^{\dagger}$ Abbreviations: AA, anhydrous ammonia; ae, acid equivalent; ai, active ingredient; AN, ammonium nitrate; COC, crop oil concentrate; MSO, Methylated seed oil; NA, none applied; and UAN, urea ammonium nitrate.

$\$$ Chemical Names: acetochlor, 2-chloro-N-(ethoxymethyl)-N-(2-ethyl-6-methylphenyl)acetamide; atrazine, 1-Chloro-3-ethylamino-5-isopropylamino-2,4,6-triazine; azoxystrobin, Methyl (2E)-2-(2-\{[6-(2-cyanophenoxy) pyrimidin-4-yl]oxy\} phenyl)-3-methoxyacrylate; dimethenameid-P, 2-chloro-N-(2,4-dimethylthiophen-3-yl)-N[(2S)-1-methoxypropan-2-yl]acetamide; glyphosate, N-(phosphonomethyl)glycine; lambda-cyhalothrin, 3-(2-chloro-3,3,3-trifluoro-1-propenyl)-2,2-dimethyl-cyano(3-phenoxyphenyl)methyl cyclopropanecarboxylate; S-metolachlor, 2-chloro-N-(2-ethyl-6-methylphenyl)-N-[(2S)-1-methoxypropan-2-yl]acetamide; nitrapyrin, 2-chloro-6-(trichloromethyl)pyridine; simazine, 6-chloro-2-N,4-N-diethyl-1,3,5-triazine-2,4-diamine; and sulflufenacil, 2-chloro-4-fluoro-5-[3-methyl-2,6-dioxo-4-(trifluoromethyl)pyrimidin-1-yl]-N-[methyl(propan2-yl)sulfamoyl]benzamide. 
Table 3. Field management information for soybean sites following corn fertilizer treatments at Novelty (2012-2015) and Albany (2014-2015)

\begin{tabular}{|c|c|c|c|c|c|c|}
\hline \multirow{2}{*}{ Management information ${ }^{\dagger}$} & \multirow{2}{*}{2012 Novelty } & \multirow{2}{*}{2013 Novelty } & \multicolumn{2}{|l|}{2014} & \multicolumn{2}{|c|}{2015} \\
\hline & & & Novelty & Albany & Novelty & Albany \\
\hline Plot size $(\mathrm{cm})$ & 3 by 12 & 3 by 12 & 3 by 15 & 3 by 10 & 3 by 10 & 3 by 15 \\
\hline Hybrid or cultivar & Ag3730 & Morsoy LL 3759N & Stine 38LE02 & AG 3731 & Stine 38LEO2 & Asgrow 3934 \\
\hline Planting date & 25 Apr. & 17 May & 8 May & 15 May & $2 \mathrm{Jul}$. & $2 \mathrm{Jul}$. \\
\hline Row spacing (cm) & 38 & 19 & 20 & 76 & 19 & 76 \\
\hline Seeding rate (seeds ha ${ }^{-1}$ ) & 444,800 & 395,400 & 444,800 & 385,500 & 469,000 & 370,000 \\
\hline Harvest date & 9 Oct. & 10 Oct. & 18 Oct. & 27 Oct. & 20 Oct. & 12 Nov. \\
\hline Tillage & No-till & No-till & No-till & Minimum & No-till & No-till \\
\hline \multicolumn{7}{|l|}{ Weed management } \\
\hline Burndown/PRE & $\begin{array}{l}25 \text { Apr., saflufenacil } \\
0.035 \mathrm{~kg} \text { ai ha }{ }^{-1}+ \\
0.25 \% \mathrm{v} / \mathrm{v} \mathrm{NIS}+ \\
\text { UAN } 2.5 \mathrm{~L} \mathrm{ha}^{-1}+ \\
\text { glyphosate } 1.2 \mathrm{~kg} \text { ae } \\
\text { ha }^{-1}\end{array}$ & $\begin{array}{l}17 \text { May, saflufenacil } \\
0.035 \mathrm{~kg} \text { ai ha }{ }^{-1}+ \\
\text { glyphosate } 1.2 \mathrm{~kg} \mathrm{ae}^{-1} \\
\mathrm{ha}^{-1}+\text { UAN } 2.5 \mathrm{~L} \mathrm{ha}^{-1}+ \\
\text { MSO } 1 \% \mathrm{v} / \mathrm{v}\end{array}$ & $\begin{array}{l}23 \text { May glufosinate } 0.8 \\
\mathrm{~kg}^{-1} \mathrm{ha}^{-1}+\text { AMS } 18 \mathrm{~g} \\
\mathrm{~L}^{-1}\end{array}$ & $\begin{array}{l}15 \text { May, } \\
\text { S-metolachlor } \\
2.2 \mathrm{~kg} \mathrm{ai} \mathrm{ha}^{-1}+ \\
\text { metribuzin } \\
\text { O.53 kg ai ha }\end{array}$ & $\begin{array}{l}3 \text { Apr., duflufenacil } \\
0.3 \mathrm{~kg} \text { ai ha }{ }^{-1}+ \\
\text { dimethenamid-P } \\
0.17 \mathrm{~kg}^{-1} \mathrm{ha}^{-1}+ \\
\text { glyphosate } 0.87 \mathrm{~kg} \\
\text { ae ha }{ }^{-1}+0.25 \% \mathrm{v} / \mathrm{v} \\
\text { NIS+ AMS } 18 \mathrm{~g} \mathrm{~L}^{-1}\end{array}$ & $\begin{array}{l}\text { S-metolachor } \\
1.5 \mathrm{~kg}^{-} \text {ai }^{-1}+ \\
\text { metribuzin } 0.4 \\
\mathrm{~kg}^{-1} \text { ai ha }\end{array}$ \\
\hline Postemergence & $\begin{array}{l}24 \text { May, fomesafen } \\
0.35 \mathrm{~kg} \mathrm{ha}^{-1}+ \\
\text { glyphosate } 1.2 \mathrm{~kg} \mathrm{ae}^{-1} \\
\mathrm{ha}^{-1}+\text { UAN } 2.3 \mathrm{~L} \mathrm{ha}^{-1} \\
+0.25 \% \mathrm{v} / \mathrm{v} \text { NIS }\end{array}$ & $\begin{array}{l}4 \text { June, glufosinate } 0.8 \\
\mathrm{~kg} \text { ai ha }{ }^{-1}+\text { AMS } 18 \mathrm{~g} \\
\mathrm{~L}^{-1} \\
1 \text { July, glufosinate } 0.8 \\
\mathrm{~kg}^{2} \text { ai ha }{ }^{-1}+ \\
\mathrm{S}-\text { metolachor } 1.36 \mathrm{~kg} \mathrm{ai} \\
\mathrm{ha}^{-1}+\text { fomesafen } 0.3 \mathrm{~kg} \\
\text { ai ha }^{-1}+\text { AMS } 18 \mathrm{~g} \mathrm{~L}^{-1} \\
+0.25 \% \mathrm{v} / \mathrm{v} \text { NIS }\end{array}$ & $\begin{array}{l}25 \text { May S-metolachor } \\
1.36 \mathrm{~kg} \text { ai ha }{ }^{-1}+ \\
\text { fomesafen } 0.3 \mathrm{~kg} \text { ai ha }^{-1} \\
+ \text { AMS } 18 \mathrm{~g} \mathrm{~L}^{-1}+ \\
0.25 \% \mathrm{v} / \mathrm{v} \text { NIS }\end{array}$ & $\begin{array}{l}3 \text { June, } \\
\text { glyphosate } 1.2 \\
\mathrm{~kg} \mathrm{ae} \mathrm{ha}^{-1}\end{array}$ & $\begin{array}{l}\text { 6 Jul., glyphosate } \\
1.58 \mathrm{~kg} \text { ae ha } \mathrm{ha}^{-1}+ \\
\text { fomesafen } 0.3 \mathrm{~kg} \text { ai } \\
\mathrm{ha}^{-1}+\mathrm{S}-\text { metolachor } \\
1.2 \mathrm{~kg} \mathrm{ai} \mathrm{ha}^{-1}\end{array}$ & $\begin{array}{l}\text { Glyphosate } \\
1.26 \mathrm{~kg} \text { ae ha }\end{array}$ \\
\hline & $\begin{array}{l}\text { 22 June, glyphosate } \\
1.2 \mathrm{~kg} \mathrm{ae} \mathrm{ha}^{-1}+\mathrm{AMS} \\
18 \mathrm{~g} \mathrm{~L}^{-1}+0.25 \% \mathrm{v} / \mathrm{v} \\
\text { NIS }\end{array}$ & & $\begin{array}{l}9 \text { July, glufosinate } 0.8 \\
\mathrm{~kg} \mathrm{ha}^{-1}+ \\
\text { flumiclorac-pentyl } 0.48 \\
\mathrm{~kg} \mathrm{ai} \mathrm{ha}^{-1}+\text { AMS } 18 \mathrm{~g} \\
\mathrm{~L}^{-1}+0.25 \% \mathrm{v} / \mathrm{v} \text { NIS }\end{array}$ & & & \\
\hline Insect management & NA & NA & NA & NA & NA & NA \\
\hline Disease management & NA & NA & $\begin{array}{l}10 \mathrm{July} \text {, azoxystrobin } \\
0.08 \mathrm{~kg} \text { ai ha } \mathrm{h}^{-1}\end{array}$ & NA & NA & NA \\
\hline
\end{tabular}

Note. ${ }^{\dagger}$ Abbreviations: ae, acid equivalent; a.i., active ingredient; COC, crop oil concentrate; MSO, Methylated seed oil; NIS, non-ionic surfactant; NA, none applied; and UAN, urea ammonium nitrate.

* Chemical name: azoxystrobin, Methyl (2E)-2-(2-\{[6-(2-cyanophenoxy)pyrimidin-4-yl]oxy\}phenyl)-3methoxyacrylate; flumiclorac-pentyl, pentyl 2-[2-chloro-5-(1,3-dioxo-4,5,6,7-tetrahydroisoindol-2-yl)-4fluorophenoxy]acetate; dimethenameid-P, 2-chloro-N-(2,4-dimethylthiophen-3-yl)-N-[(2S)-1-methoxypropan -2-yl]acetamide; fomesafen, 5-[2-chloro-4-(trifluoromethyl)phenoxy]-N-methylsulfonyl-2-nitrobenzamide; glufosinate, 2-amino-4-[hydroxy(methyl)phosphoryl]butanoic acid; glyphosate, N-(phosphonomethyl)glycine; metrabuzin, 4-amino-6-tert-butyl-3-methylsulfanyl-1,2,4-triazin-5-one; S-metolachlor, 2-chloro-N-(2-ethyl-6 -methylphenyl)-N-[(2S)-1-methoxypropan-2-yl]acetamide; and sulflufenacil, 2-chloro-4-fluoro-5-[3-methyl2,6-dioxo-4-(trifluoromethyl)pyrimidin-1-yl]-N-[methyl(propan-2-yl)sulfamoyl]benzamide.

In 2014, upstate Missouri experienced record yields due not only to cool summer temperatures (data not presented), but also to uniform distribution of precipitation, with Novelty receiving $771 \mathrm{~mm}$ and Albany receiving $751 \mathrm{~mm}$. In 2015, precipitation at Albany and Novelty was above average, which delayed soybean planting.

Initial soil samples were taken from each site (Table 1). Soil test P levels were very low (Novelty in 2011, 2012), low (Novelty in 2013, 2014, and Albany in 2013), and medium (Albany in 2014) according to Buchholz et al. (2004). Soil test Zn levels were low (Novelty in 2011, 2012, and 2013) or medium (Novelty in 2014, Albany in 2013 and 2014) (Buchholz, Brown, Garret, Hanson, \& Wheaton, 2004). Finally, soil test S was medium across all site-years (Buchholz et al., 2004). 


\subsection{Corn Response}

Corn plant population was similar at all site-years (Novelty in 2011 and 2012, $P=0.86$, and Novelty and Albany in 2013 and 2014, $P=0.92$ ). Ear leaf tissue concentration at VT for $\mathrm{P}, \mathrm{S}$, and $\mathrm{Zn}$ were combined over the two years (Novelty in 2013 and 2014) where the measurements were collected due to an absence of a significant interaction between years. These sites had low soil test $\mathrm{P}$ and low-to-medium soil test $\mathrm{Zn}$. Ear leaf $\mathrm{P}$ concentrations ranged from 2.38 to $3.13 \mathrm{~g} \mathrm{~kg}^{-1}$ (Table 4). All treatments, except the non-treated control, were similar and were within the sufficiency range for ear leaf $P$ concentrations ( 2.5 to $5.0 \mathrm{~g} \mathrm{~kg}^{-1}$ ) (Jones et al., 1967; Bryson et al., 2014). The non-treated control had $2.4 \mathrm{~g} \mathrm{~kg}^{-1} \mathrm{P}$, which was below the plant sufficiency range (Jones et al., 1967; Bryson et al., 2014). Concentrations of ear leaf S and ear leaf P were similar. All treatments were similar, and they were up to $0.2 \mathrm{~g} \mathrm{~kg}^{-1}$ greater than the non-treated no $\mathrm{N}$ or no $\mathrm{P}$ controls. All treatments including $\mathrm{P}$ and $\mathrm{N}$ were within the ear leaf sufficiency range $\left(1.5\right.$ to $4.0 \mathrm{~g} \mathrm{~kg}^{-1}$ ) for $\mathrm{Zn}$ (Jones, 1967; Bryson et al., 2014). Ear leaf $S$ concentrations ranged from 1.6 to $2.1 \mathrm{~g} \mathrm{~kg}^{-1}$ and were greater than the no $\mathrm{N}$ and no $\mathrm{P}$ controls. Similar results for ear leaf $\mathrm{P}$ and $\mathrm{S}$ concentrations were reported in Iowa (Sawyer \& Barker, 2010). There was no clear effect of $\mathrm{Zn}$ on P concentration, similar to other research evaluating P-Zn interactions (Keefer et al., 1972; Rehm et al., 1981).

Table 4. Corn ear $\mathrm{SO}_{4}-\mathrm{S}$ concentration for $\mathrm{Zn}$ treatments at Novelty from 2013-2014. Data were combined over years. All $\mathrm{P}$ amounts were $80 \mathrm{~kg} \mathrm{P}_{2} \mathrm{O}_{5}$ ha $^{-1}$

\begin{tabular}{|c|c|c|c|c|}
\hline${\mathrm{Zn} \text { treatments }{ }^{\dagger}}^{\dagger}$ & $\mathrm{Zn}$ amount & $\mathrm{P}$ & $\mathrm{Zn}$ & $\mathrm{SO}_{4}-\mathrm{S}$ \\
\hline & ---- $\mathrm{kg} \mathrm{ha}^{-1}$---- & ---- $\mathrm{g} \mathrm{kg}^{-1}$---- & ---- $\mathrm{mg} \mathrm{kg}^{-1}$ & ---- $\mathrm{g} \mathrm{kg}^{-1}$ \\
\hline Non-treated control & 0 & 2.4 & 20.9 & 1.7 \\
\hline $\mathrm{N}$ only control & 0 & 2.9 & 30.2 & 2.1 \\
\hline DAP & 0 & 2.8 & 28.6 & 2.0 \\
\hline $\mathrm{DAP}+\mathrm{ZnSO}_{4}$ & 2.2 & 3.0 & 29.4 & 2.1 \\
\hline $\mathrm{DAP}+\mathrm{ZnSO}_{4}$ & 5.6 & 3.0 & 30.4 & 2.1 \\
\hline MAP & 0 & 3.0 & 28.5 & 2.1 \\
\hline $\mathrm{MAP}+\mathrm{ZnSO}_{4}$ & 2.2 & 2.9 & 27.5 & 2.0 \\
\hline $\mathrm{MAP}+\mathrm{ZnSO}_{4}$ & 5.6 & 2.8 & 27.7 & 1.9 \\
\hline MES10 & 0 & 2.8 & 26.1 & 1.9 \\
\hline MESZ & 2.2 & 2.9 & 27.1 & 2.0 \\
\hline $\mathrm{MAP}+\mathrm{AS}$ & 0 & 3.1 & 27.7 & 2.1 \\
\hline MAP + SuperZn & 2.2 & 3.1 & 27.7 & 2.0 \\
\hline MAP + SuperZn & 5.6 & 3.0 & 29.1 & 2.0 \\
\hline $\mathrm{DAP}+\mathrm{AS}$ & 0 & 3.0 & 27.2 & 2.1 \\
\hline $\mathrm{DAP}+$ SuperZn & 2.2 & 3.0 & 26.5 & 2.0 \\
\hline DAP + SuperZn & 5.6 & 3.1 & 29.2 & 2.0 \\
\hline $\operatorname{LSD}(P=0.05)$ & & 0.3 & 2.9 & 0.2 \\
\hline
\end{tabular}

Note. ${ }^{\dagger}$ Abbreviations: AMS, ammonium sulfate; DAP, diammonium phosphate; MAP, monoammonium phosphate.

Ear leaf $\mathrm{Zn}$ concentrations were interesting because the N-only control treatment had the second highest $\mathrm{Zn}$ concentration, which indicated sufficient plant available $\mathrm{Zn}$ in the soil. An application of $\mathrm{DAP}+\mathrm{ZnSO}_{4}$ at $5.6 \mathrm{~kg}$ $\mathrm{Zn} \mathrm{ha}^{-1}$ and N-only treatments had 4.13 to $9.43 \mathrm{mg} \mathrm{kg}^{-1}$ greater ear leaf $\mathrm{Zn}$ concentration than MES10 and the non-treated control. The non-treated, no $\mathrm{N}$ control was 5.15 to $9.43 \mathrm{mg} \mathrm{kg}^{-1}$ less than all other treatments for ear leaf $\mathrm{Zn}$ concentration, but this was probably due to $\mathrm{N}$ affecting $\mathrm{Zn}$ uptake. These data indicate no clear impact of the $\mathrm{Zn}$ treatments on ear leaf $\mathrm{Zn}$ concentration over the two years ear leaves were collected since the $\mathrm{N}$-only control had ear leaf $\mathrm{Zn}$ concentrations similar to the $\mathrm{Zn}$ treatments. A corn fertilizer study in West Virginia with three amounts of $\mathrm{Zn}\left(0,3.36\right.$, and $\left.6.72 \mathrm{~kg} \mathrm{Zn} \mathrm{ha}^{-1}\right)$ also observed no-yield response to $\mathrm{Zn}$ treatment (Stout \& Bennett, 1983). An efficacy study of $\mathrm{ZnO}$ concluded that it and $\mathrm{ZnSO}_{4}$ had similar plant nutrient recovery when incorporated in the soil; however, $\mathrm{ZnSO}_{4}$ had a greater plant recovery than $\mathrm{ZnO}$ when the fertilizer was band- or surface-applied (McBeath \& McLaughlin, 2014). In Michigan, a three-year study with $220 \mathrm{~kg}$ of fertilizer ha ${ }^{-1}$ comparing MAP and DAP observed no change in tissue $\mathrm{P}$ or $\mathrm{Zn}$ concentration and detected no yield difference 
between MAP and DAP (Yerokun \& Christenson, 1990). In addition, P fertility did not appear to decrease $\mathrm{Zn}$ uptake, similar to Halim et al. (1968).

A significant two-way interaction occurred between treatments and site-years for corn grain yield; therefore, data were analyzed by individual site-years and reported separately (Table 5). In a planned comparison, contrasts comparing $\mathrm{Zn}$ amounts (2.2 vs $5.6 \mathrm{~kg} \mathrm{Zn} \mathrm{ha}^{-1}$ ), $\mathrm{Zn}$ sources (SuperZn vs. $\mathrm{ZnSO}_{4}$ ), and P sources (MAP vs. DAP) showed no significant differences in yield for the four site-years (Novelty and Albany in 2014 and 2015) that were evaluated (data not presented). At Novelty in 2011, all treatments yielded 6,670 to 7,860 kg ha-1 greater than the non-treated, no-N control with yields from 9,090 to $10,280 \mathrm{~kg} \mathrm{ha}^{-1}$ (Table 5). With the extreme drought in 2012, yields were low $\left(1,120\right.$ to $\left.1,770 \mathrm{~kg} \mathrm{ha}^{-1}\right)$. The non-treated control, N-only, and MESZ yielded 531 to 643 $\mathrm{kg} \mathrm{ha}^{-1}$ greater than MAP, MAP+ZnSO 4 at $2.2 \mathrm{~kg} \mathrm{Zn} \mathrm{ha}^{-1}, \mathrm{MAP}^{2} \mathrm{ZnSO}_{4}$ at $5.6 \mathrm{~kg} \mathrm{Zn} \mathrm{ha}^{-1}$, and MAP+AMS. At Novelty in 2013, MAP+SuperZn at $5.6 \mathrm{~kg} \mathrm{Zn} \mathrm{ha}^{-1}$ and DAP+AMS increased yields 560 to 2,850 over the non-treated control, $\mathrm{N}$-only control, $\mathrm{DAP}+\mathrm{ZnSO}_{4}$ at $5.6 \mathrm{~kg} \mathrm{Zn} \mathrm{ha}^{-1}$, and $\mathrm{DAP}+\mathrm{ZnSO}_{4}$ at $2.2 \mathrm{~kg} \mathrm{Zn} \mathrm{ha}^{-1}$.

Table 5. Corn grain yield response to Zn treatments at Novelty (2011-2014) and Albany (2013-2014). Phosphorus was applied at $90 \mathrm{~kg} \mathrm{P}_{2} \mathrm{O}_{5}$ ha $^{-1}$

\begin{tabular}{|c|c|c|c|c|c|c|c|}
\hline \multirow{2}{*}{$\mathrm{Zn}$ treatments ${ }^{\dagger}$} & \multirow{2}{*}{ Zn amount } & \multicolumn{4}{|c|}{ Novelty } & \multicolumn{2}{|c|}{ Albany } \\
\hline & & 2011 & 2012 & 2013 & 2014 & 2013 & 2014 \\
\hline & ------------ & - & - & $\mathrm{kg} \mathrm{ha}{ }^{-}$ & 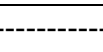 & ------ & - n---- \\
\hline Non-treated control & 0.0 & 2,420 & 1,770 & 6,800 & 12,700 & 6,520 & 7,240 \\
\hline $\mathrm{N}$ only control & 0.0 & 9,090 & 1,710 & 9,080 & 16,180 & 6,990 & 8,970 \\
\hline DAP & 0.0 & 9,420 & 1,380 & 9,420 & 15,950 & 6,930 & 9,200 \\
\hline $\mathrm{DAP}+\mathrm{ZnSO}_{4}$ & 2.2 & 9,230 & 1,600 & 9,040 & 16,800 & 6,520 & 10,420 \\
\hline $\mathrm{DAP}+\mathrm{ZnSO}_{4}$ & 5.6 & 9,480 & 1,620 & 9,010 & 15,820 & 6,520 & 8,760 \\
\hline MAP & 0.0 & 9,740 & 1,180 & 9,200 & 16,480 & 6,590 & 10,540 \\
\hline $\mathrm{MAP}+\mathrm{ZnSO}_{4}$ & 2.2 & 10,270 & 1,120 & 9,620 & 16,340 & 6,590 & 9,870 \\
\hline $\mathrm{MAP}+\mathrm{ZnSO}_{4}$ & 5.6 & 9,660 & 1,130 & 9,470 & 16,800 & 6,660 & 10,420 \\
\hline MES10 & 0.0 & 9,890 & 1,420 & 9,500 & 16,710 & 6,590 & 8,810 \\
\hline MESZ & 2.2 & 10,280 & 1,750 & 9,610 & 16,130 & 6,790 & 7,210 \\
\hline $\mathrm{MAP}+\mathrm{AS}$ & 0.0 & 9,800 & 1,120 & 9,350 & 16,140 & 6,660 & 8,710 \\
\hline MAP + SuperZn & 2.2 & $-*$ & - & 9,500 & 16,280 & 7,260 & 10,200 \\
\hline MAP + SuperZn & 5.6 & - & - & 9,650 & 16,730 & 6,660 & 10,230 \\
\hline $\mathrm{DAP}+\mathrm{AS}$ & 0.0 & - & - & 9,640 & 16,490 & 6,660 & 9,470 \\
\hline DAP + SuperZn & 2.2 & - & - & 9,510 & 14,790 & 6,660 & 10,220 \\
\hline DAP + SuperZn & 5.6 & - & - & 9,400 & 16,580 & 6,930 & 8,560 \\
\hline $\operatorname{LSD}(P=0.05)$ & & 1,260 & 450 & 540 & 806 & NS & 1,580 \\
\hline
\end{tabular}

Note. ${ }^{\dagger}$ Abbreviations: AMS, ammonium sulfate; DAP, diammonium phosphate; MAP, monoammonium phosphate.

${ }^{\ddagger}$ Treatments were not applied at these two locations.

All other treatments had similar grain yields. Grain yields at Albany in 2013 were similar among treatments $(P=$ 0.57). In 2014, both Novelty and Albany experienced exceptionally high yields $\left(12,700\right.$ to $16,800 \mathrm{~kg} \mathrm{ha}^{-1}$ and 7,210 to $10,540 \mathrm{~kg} \mathrm{ha}^{-1}$, respectively), due to good overall precipitation (Figure 1) and low temperatures during pollination and grain fill (data not presented). At Novelty, $\mathrm{DAP}+\mathrm{ZnSO}_{4}$ at $2.2 \mathrm{~kg} \mathrm{Zn} \mathrm{ha}{ }^{-1}, \mathrm{MAP}+\mathrm{ZnSO}_{4}$ at $5.6 \mathrm{~kg}$ $\mathrm{Zn} \mathrm{ha}{ }^{-1}$, MES10, and MAP+SuperZn at $5.6 \mathrm{~kg} \mathrm{Zn} \mathrm{ha}^{-1}$ had 763 to $4098 \mathrm{~kg} \mathrm{ha}^{-1}$ greater yields than the non-treated control, DAP, DAP+ZnSO ${ }_{4}$ at $5.6 \mathrm{~kg} \mathrm{Zn} \mathrm{ha}^{-1}$, or DAP+SuperZn at $2.2 \mathrm{~kg} \mathrm{Zn} \mathrm{ha}^{-1}$. At Albany in 2014, MAP alone yielded 1,570 to 3,300 $\mathrm{kg} \mathrm{ha}^{-1}$ greater than the non-treated control, N-only, MES10, MESZ, DAP+SuperZn at $5.6 \mathrm{~kg} \mathrm{Zn} \mathrm{ha}^{-1}, \mathrm{DAP}+\mathrm{ZnSO}_{4}$ at $5.6 \mathrm{~kg} \mathrm{Zn} \mathrm{ha}^{-1}$, and MAP+AMS. Albany had medium soil test $\mathrm{P}, \mathrm{S}$, and $\mathrm{Zn}$, which likely resulted in limited yield differences among treatments (Table 1). An increase in corn grain yield has been related to $\mathrm{P}$ uptake which has been greater than Zn fertility (Rehm et al., 1983), and there was no apparent Zn-P interaction which was similar to other research (Rehm et al., 1981). 
Table 6. Corn grain protein concentrations at Novelty (2011-2014) and Albany (2013-2014). Data combined over years were denoted. Phosphorus was applied at $90 \mathrm{~kg} \mathrm{P}_{2} \mathrm{O}_{5} \mathrm{ha}^{-1}$

\begin{tabular}{|c|c|c|c|c|}
\hline $\mathrm{Zn}_{\text {treatments }}{ }^{\dagger}$ & $\mathrm{Zn}$ amount & Novelty 2011 & Novelty 2013 and $2014^{\ddagger}$ & Albany 2013 and $2014^{\ddagger}$ \\
\hline & ---- $\mathrm{kg} \mathrm{ha}^{-1}$---- & - & $\mathrm{g} \mathrm{kg}^{-1}$ & \\
\hline Non-treated control & 0 & 70 & 69 & 73 \\
\hline $\mathrm{N}$ only control & 0 & 94 & 84 & 75 \\
\hline DAP & 0 & 96 & 85 & 79 \\
\hline $\mathrm{DAP}+\mathrm{ZnSO}_{4}$ & 5.6 & 96 & 85 & 80 \\
\hline $\mathrm{DAP}+\mathrm{ZnSO}_{4}$ & 2.2 & 95 & 85 & 80 \\
\hline MAP & 0 & 89 & 84 & 77 \\
\hline $\mathrm{MAP}+\mathrm{ZnSO}_{4}$ & 5.6 & 94 & 84 & 83 \\
\hline $\mathrm{MAP}+\mathrm{ZnSO}_{4}$ & 2.2 & 96 & 85 & 75 \\
\hline MES10 & 0 & 95 & 84 & 77 \\
\hline MESZ & 2.2 & 94 & 83 & 81 \\
\hline $\mathrm{MAP}+\mathrm{AS}$ & 0 & 95 & 85 & 82 \\
\hline MAP + SuperZn & 2.2 & - & 84 & 82 \\
\hline MAP + SuperZn & 5.6 & - & 83 & 82 \\
\hline $\mathrm{DAP}+\mathrm{AS}$ & 0 & - & 83 & 83 \\
\hline DAP + SuperZn & 2.2 & - & 83 & 80 \\
\hline DAP + SuperZn & 5.6 & - & 83 & 83 \\
\hline $\operatorname{LSD}(P=0.05)$ & & 7 & 2 & 5 \\
\hline
\end{tabular}

Note. ${ }^{\dagger}$ Abbreviations: AMS, ammonium sulfate; DAP, diammonium phosphate; MAP, monoammonium phosphate.

${ }^{\ddagger}$ Data were combined over years.

$\S$ Treatments were not applied during these two years.

Grain oil, protein, and starch concentrations were observed at Novelty (2011, 2013, and 2014) and Albany (2013 and 2014), but none of the treatments affected grain oil concentration at any site-year [(Novelty in 2011 and 2012, $P=0.41$ ) (Novelty and Albany in 2013 and 2014, $P=0.16$ )] (data not presented). Grain protein at Novelty in 2013 and 2014 as well as Albany in 2013 and 2014 had no treatment-by-year interaction, so data were combined over years (Table 6). At Novelty in 2011, protein concentration ranged from 70 to $96 \mathrm{~g} \mathrm{~kg}^{-1}$. All fertilizer treatments were similar, but they were 19 to $26 \mathrm{~g} \mathrm{~kg}^{-1}$ greater than the no $\mathrm{N}$, non-treated control. Novelty in 2012 showed no significant difference $(P=0.11)$ among treatments, which likely was due to extreme drought (data not presented). At Novelty and Albany in 2013 and 2014, protein concentration with MAP+AMS was significantly greater than MAP+SuperZn at $5.6 \mathrm{~kg} \mathrm{Zn} \mathrm{ha}^{-1}$ and the non-treated control by 3 and $15 \mathrm{~g} \mathrm{~kg}^{-1}$, respectively. All other fertilized treatments had similar protein concentrations that ranged from 83 to $85 \mathrm{mg} \mathrm{kg}^{-1}$. At Albany in 2013 and 2014, protein concentrations ranged from 73 to $83 \mathrm{~g} \mathrm{~kg}^{-1}$. DAP+AMS, MAP+ZnSO 4 at $5.6 \mathrm{~kg} \mathrm{Zn} \mathrm{ha}^{-1}$, and DAP+SuperZn at $5.6 \mathrm{~kg} \mathrm{Zn} \mathrm{ha}^{-1}$ had 6 to $11 \mathrm{~g} \mathrm{~kg}^{-1}$ greater protein concentration than the non-treated control, N-only, MAP, MES10, and MAP $+Z_{n S O}$ at $2.2 \mathrm{~kg} \mathrm{Zn} \mathrm{ha}^{-1}$. At Novelty in 2011, the non-treated control had 64 to $170 \mathrm{~g} \mathrm{~kg}^{-1}$ higher starch concentration than all other treatments (Table 7). MAP had 68 to $124 \mathrm{~g} \mathrm{~kg}^{-1}$ greater starch concentration than all other fertilizer treatments. Novelty in 2012 showed no differences among treatments $(P=0.87)$. At Novelty in 2013 and 2014, the non-treated control was 52 to $96 \mathrm{~g}$ $\mathrm{kg}^{-1}$ greater than all treatments except DAP+AMS. At Albany in 2013 and 2014, DAP, DAP+ZnSO 4 at $5.6 \mathrm{~kg} \mathrm{Zn}$ $\mathrm{ha}^{-1}, \mathrm{MAP}+\mathrm{ZnSO}_{4}$ at $2.2 \mathrm{~kg} \mathrm{Zn} \mathrm{ha}^{-1}$, and DAP+SuperZn at $2.2 \mathrm{~kg} \mathrm{Zn} \mathrm{ha}^{-1}$ had 59 to $100 \mathrm{~g} \mathrm{~kg}^{-1}$ greater grain starch concentration than MAP+SuperZn at $2.2 \mathrm{~kg} \mathrm{Zn} \mathrm{ha}^{-1}$, DAP+SuperZn at $5.6 \mathrm{~kg} \mathrm{Zn} \mathrm{ha}{ }^{-1}$, DAP+SuperZn at $2.2 \mathrm{~kg} \mathrm{Zn} \mathrm{ha}^{-1}$. At Albany in 2013 and 2014, all treatments had similar starch concentrations $(P=0.17)$. Although significance occurred at five site-years for protein and three site-years for starch concentrations, differences were inconsistent. The relationship between nitrogen and corn protein concentration is commonly observed (Uribelarrea, Below, \& Moose, 2004); however, Kaiser and Lamb (2008) showed protein contents were lower when $\mathrm{P}$ was applied, compared to no $\mathrm{P}$ application, especially at $120 \mathrm{~kg} \mathrm{~N}^{-1}$ and greater $\mathrm{N}$ applications. However, we did not observe this in our research. 


\subsection{Soil Test P, S, and Zn Following Corn}

Treatments with applied $\mathrm{P}$ fertilizer had 16 to $37 \mathrm{~kg} \mathrm{ha}^{-1}$ greater soil test $\mathrm{P}$ than the non-treated control and the $\mathrm{N}$-only treatment (Table 8). The largest soil test $\mathrm{P}$ was DAP+SuperZn at $5.6 \mathrm{~kg} \mathrm{Zn} \mathrm{ha}^{-1}$, which had 16 to $33 \mathrm{~kg}$ $\mathrm{ha}^{-1}$ greater soil test $\mathrm{P}$ than the non-treated control, N-only, MAP, DAP, DAP+SuperZn at $2.2 \mathrm{~kg} \mathrm{Zn} \mathrm{ha}^{-1}$, and MAP+SuperZn at $2.2 \mathrm{~kg} \mathrm{Zn} \mathrm{ha}^{-1}$. However, the DAP treatment had $14.8 \mathrm{~kg} \mathrm{ha}^{-1}$ less soil test $\mathrm{P}$ than $\mathrm{MAP}+\mathrm{SuperZn}$ at $5.6 \mathrm{~kg} \mathrm{Zn} \mathrm{ha}{ }^{-1}$, while all other treatments with $\mathrm{P}$ had similar soil test $\mathrm{P}$.

Table 7. Grain starch concentrations at Novelty (2011, 2013-2014) and Albany (2013-2014). Data were combined over years were denoted. Phosphorus was applied at $90 \mathrm{~kg} \mathrm{P}_{2} \mathrm{O}_{5} \mathrm{ha}^{-1}$ for all treatments including $\mathrm{P}$

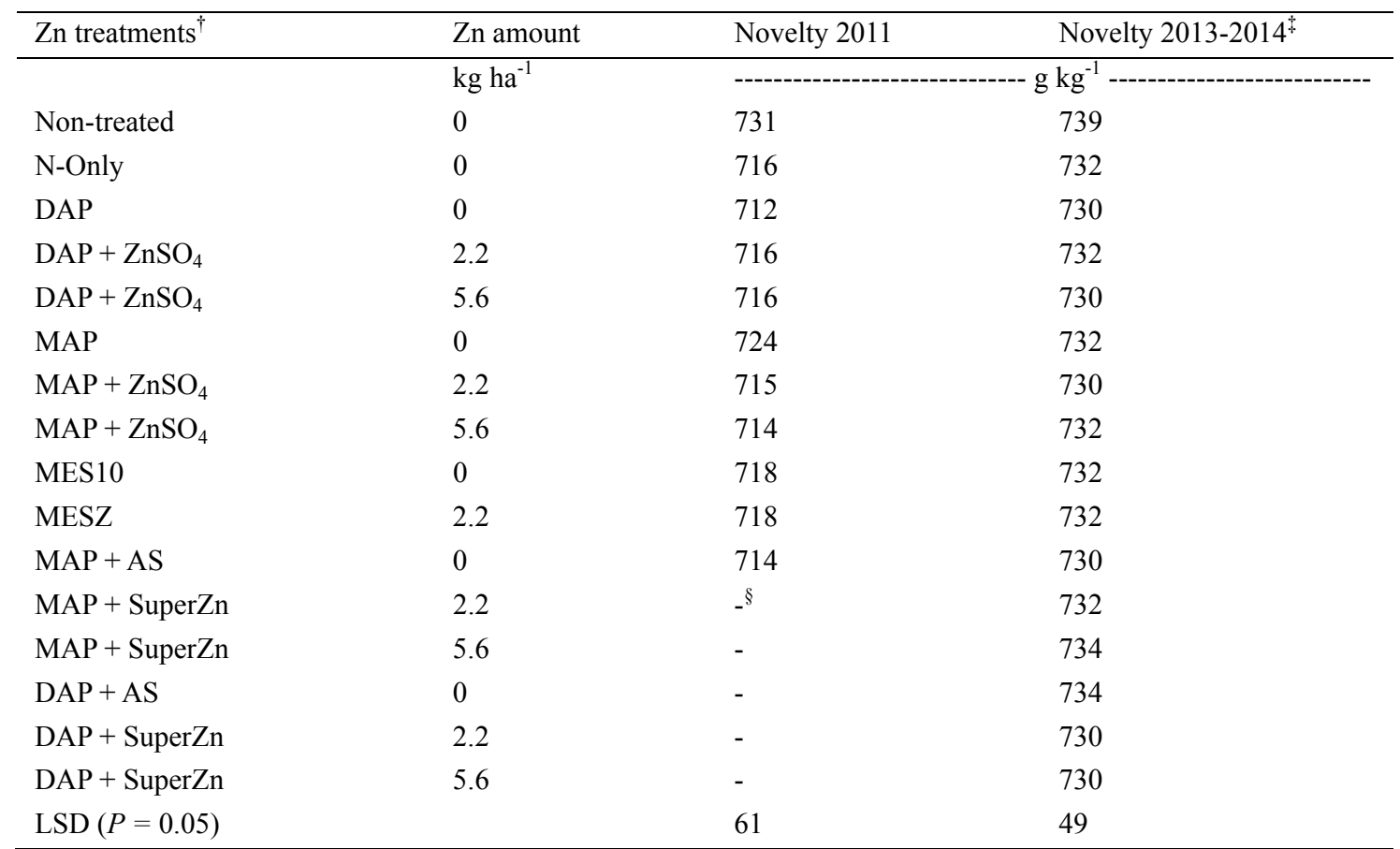

Note. ${ }^{\dagger}$ Abbreviations: AMS, ammonium sulfate; DAP, diammonium phosphate; MAP, monoammonium phosphate.

${ }^{\ddagger}$ Data were combined over years. No differences among treatments were observed in 2012.

${ }^{\S}$ Treatments were not applied during these two years. 
Table 8. Soil test $\mathrm{P}, \mathrm{Zn}$, and $\mathrm{SO}_{4}$-S after corn harvest at Novelty and Albany (2013-2014). Phosphorus was applied at $90 \mathrm{~kg} \mathrm{P}_{2} \mathrm{O}_{5}$ ha $^{-1}$ for all treatments including $\mathrm{P}$

\begin{tabular}{|c|c|c|c|c|c|c|}
\hline \multirow[b]{2}{*}{$\mathrm{Zn}$ treatments ${ }^{\dagger}$} & \multirow[b]{2}{*}{$\mathrm{Zn}$ amount } & \multirow[b]{2}{*}{$\mathrm{P}^{\ddagger}$} & \multicolumn{2}{|c|}{$\mathrm{Zn}$} & \multicolumn{2}{|c|}{$\mathrm{SO}_{4}-\mathrm{S}$} \\
\hline & & & $\begin{array}{l}\text { Novelty } \\
2013 \text { and } 2014\end{array}$ & $\begin{array}{l}\text { Albany } \\
2013 \text { and } 2014\end{array}$ & $\begin{array}{l}\text { Novelty } \\
2013 \text { and } 2014\end{array}$ & $\begin{array}{l}\text { Albany } \\
2013 \text { and } 2014\end{array}$ \\
\hline & --- $\mathrm{kg} \mathrm{ha}^{-1}$ & $\mathrm{~kg} \mathrm{ha}^{-1}$ & ----- mg ha ${ }^{-1}$----- & ----- mg ha ${ }^{-1}$ & ----- mg ha ${ }^{-1}$----- & ----- mg ha ${ }^{-1}$ \\
\hline Non-treated & 0 & 33.8 & 0.58 & 0.80 & 4.48 & 5.40 \\
\hline N-Only & 0 & 31.6 & 0.56 & 0.70 & 4.08 & 4.19 \\
\hline DAP & 0 & 48.3 & 0.61 & 1.20 & 4.26 & 4.61 \\
\hline $\mathrm{DAP}+\mathrm{ZnSO}_{4}$ & 2.2 & 50.8 & 0.84 & 2.20 & 5.40 & 4.64 \\
\hline $\mathrm{DAP}+\mathrm{ZnSO}_{4}$ & 5.6 & 55.1 & 1.42 & 1.00 & 5.02 & 5.23 \\
\hline MAP & 0 & 49.4 & 0.66 & 2.00 & 4.57 & 5.88 \\
\hline $\mathrm{MAP}+\mathrm{ZnSO}_{4}$ & 2.2 & 58.7 & 1.08 & 1.30 & 4.86 & 4.69 \\
\hline $\mathrm{MAP}+\mathrm{ZnSO}_{4}$ & 5.6 & 53.3 & 2.32 & 2.00 & 5.34 & 5.48 \\
\hline MES10 & 0 & 55.4 & 0.62 & 1.40 & 6.64 & 5.24 \\
\hline MESZ & 2.2 & 56.1 & 1.08 & 1.10 & 5.55 & 4.59 \\
\hline $\mathrm{MAP}+\mathrm{AS}$ & 0 & 55.5 & 0.67 & 0.90 & 5.40 & 6.20 \\
\hline MAP + SuperZn & 2.2 & 50.2 & 0.84 & 1.10 & 4.89 & 5.33 \\
\hline MAP + SuperZn & 5.6 & 61.5 & 1.37 & 1.56 & 4.79 & 5.93 \\
\hline $\mathrm{DAP}+\mathrm{AS}$ & 0 & 53.9 & 0.67 & 0.90 & 4.76 & 5.45 \\
\hline $\mathrm{DAP}+$ SuperZn & 2.2 & 48.7 & 1.10 & 1.10 & 5.41 & 5.50 \\
\hline $\mathrm{DAP}+$ SuperZn & 5.6 & 64.8 & 2.44 & 1.50 & 4.80 & 5.13 \\
\hline LSD & & 12.9 & 0.58 & NS & 0.96 & 0.75 \\
\hline
\end{tabular}

Note. ${ }^{\dagger}$ Abbreviations: AMS, ammonium sulfate; DAP, diammonium phosphate; MAP, monoammonium phosphate.

"Data were combined over sites and years.

${ }^{\S}$ Data were combined over years.

Soil test $\mathrm{S}$ and $\mathrm{Zn}$ had a significant site-year-by-treatment interaction, but due to similar soil series, data were combined over years for individual sites and analyzed. Soil test $\mathrm{Zn}$ ranged from 0.56 to $2.44 \mathrm{mg} \mathrm{ha}^{-1}$ and 0.71 to $1.56 \mathrm{mg} \mathrm{ha}^{-1}$ at Novelty and Albany, respectively. All treatments had at least medium soil test $\mathrm{Zn}$ at the end of the growing season (Buchholz et al., 2004). When Zn was applied at Novelty in 2013 and 2014, soil test Zn generally increased compared to fertilizer treatments that had no $\mathrm{Zn}$. DAP+SuperZn at $5.6 \mathrm{~kg} \mathrm{Zn} \mathrm{ha}^{-1}$ and $\mathrm{MAP}+\mathrm{ZnSO}_{4}$ at $5.6 \mathrm{~kg} \mathrm{Zn} \mathrm{ha}{ }^{-1}$ had significantly greater soil test $\mathrm{Zn}$ than all other treatments, while all other treatments with $\mathrm{P}, \mathrm{S}$, or $\mathrm{Zn}$, were similar. At Albany, $\mathrm{DAP}+\mathrm{ZnSO}_{4}$ at $2.2 \mathrm{~kg} \mathrm{Zn} \mathrm{ha}^{-1}$ and $\mathrm{MAP}+\mathrm{ZnSO} \mathrm{Z}_{4}$ at $5.6 \mathrm{~kg}$ $\mathrm{Zn} \mathrm{ha}{ }^{-1}$, and MAP had 1.1 to $1.5 \mathrm{mg} \mathrm{kg}^{-1}$ greater soil test $\mathrm{Zn}$ than DAP+AMS, MAP+AMS, N-only, and the non-treated control, but all other treatments were similar. This indicates that even though there was no crop yield response to $\mathrm{Zn}$, soil buildup occurred. 
Table 9. Soybean plant population, grain yield, oil, and protein concentration response to fertilizer treatments applied the previous year at Novelty (2012-2015) and Albany (2014-2015)

\begin{tabular}{|c|c|c|c|c|c|c|c|c|c|}
\hline \multirow{2}{*}{$\mathrm{Zn}$ treatments ${ }^{\dagger}$} & \multirow{2}{*}{$\mathrm{Zn}$ amount } & \multicolumn{2}{|c|}{ Population } & \multicolumn{2}{|c|}{ Yield } & \multicolumn{2}{|c|}{ Oil } & \multicolumn{2}{|c|}{ Protein } \\
\hline & & $2012-13$ & $2014-15$ & $2012-13$ & $2014-15$ & $2012-13$ & 2014-15 & $2012-13$ & $2014-15$ \\
\hline & -- $\mathrm{kg} \mathrm{ha}^{-1}--$ & \multicolumn{2}{|c|}{------ plants ha ${ }^{-1}$------ } & \multicolumn{2}{|c|}{------ $\mathrm{kg} \mathrm{ha}^{-1}$------ } & \multicolumn{4}{|c|}{ - } \\
\hline Non-treated & 0 & 307,600 & 321,700 & 2,640 & 2,900 & 197 & 189 & 352 & 349 \\
\hline N-only & 0 & 314,300 & 317,000 & 2,670 & 3,120 & 193 & 189 & 357 & 348 \\
\hline DAP & 0 & 310,100 & 292,300 & 2,570 & 3,170 & 192 & 188 & 360 & 349 \\
\hline $\mathrm{DAP}+\mathrm{ZnSO} 4$ & 2.2 & 331,600 & 312,100 & 2,620 & 3,080 & 193 & 188 & 358 & 347 \\
\hline $\mathrm{DAP}+\mathrm{ZnSO} 4$ & 5.6 & 122,800 & 336,800 & 2,620 & 3,130 & 192 & 189 & 358 & 347 \\
\hline MAP & 0 & 340,300 & 321,000 & 2,550 & 3,120 & 191 & 188 & 360 & 348 \\
\hline $\mathrm{MAP}+\mathrm{ZnSO} 4$ & 2.2 & 316,500 & 333,600 & 2,590 & 3,140 & 192 & 188 & 359 & 349 \\
\hline $\mathrm{MAP}+\mathrm{ZnSO} 4$ & 5.6 & 280,000 & 309,400 & 2,600 & 3,120 & 193 & 188 & 357 & 347 \\
\hline MESZ & 2.2 & 333,600 & 333,100 & 2,623 & 3,140 & 191 & 189 & 360 & 349 \\
\hline MES10 & 0 & 340,300 & 311,600 & 2,650 & 3,130 & 193 & 188 & 357 & 346 \\
\hline $\mathrm{MAP}+\mathrm{AMS}$ & 0 & 323,000 & 302,500 & 2,660 & 3,130 & 192 & 188 & 358 & 348 \\
\hline MAP + SuperZn & 2.2 & 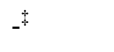 & 317,500 & - & 3,210 & - & 189 & - & 347 \\
\hline MAP + SuperZn & 5.6 & - & 299,700 & - & 3,190 & - & 189 & - & 344 \\
\hline $\mathrm{DAP}+\mathrm{AMS}$ & 0 & - & 299,700 & - & 3,230 & - & 189 & - & 346 \\
\hline $\mathrm{DAP}+$ SuperZn & 2.2 & - & 295,000 & - & 3,060 & - & 190 & - & 347 \\
\hline $\mathrm{DAP}+$ SuperZn & 5.6 & - & 333,100 & - & 3,130 & - & 189 & - & 348 \\
\hline P-value & & 0.5 & 0.15 & 0.85 & 0.16 & 0.42 & 0.37 & 0.33 & 0.46 \\
\hline
\end{tabular}

Note. ${ }^{\dagger}$ Population and yield data were determined for all site-years. Grain oil and protein data were determined at all site-years except Albany in 2014. Data were combined over site-years unless denoted otherwise.

Abbreviations: AMS, ammonium sulfate; DAP, diammonium phosphate; MAP, monoammonium phosphate.

$*$ Treatments were not applied during these two years.

Soil test S ranged from 4.1 to $6.6 \mathrm{mg} \mathrm{kg}^{-1}$ and 4.2 to $6.2 \mathrm{mg} \mathrm{kg}^{-1}$ at Novelty and Albany, respectively. All treatments had medium soil test $\mathrm{S}$. At Novelty, all treatments with $\mathrm{S}$ or $\mathrm{Zn}$ had similar soil test $\mathrm{S}$ levels (Buchholz et al., 2004). MESZ and MES10 had 0.98 to 2.56 higher soil test S amounts than the non-treated control, N-only, DAP, and MAP. At Albany, MAP+AMS had a 1.07 to $2.01 \mathrm{mg} \mathrm{kg}^{-1}$ greater soil test S concentration than DAP+SuperZn at $5.6 \mathrm{~kg} \mathrm{Zn} \mathrm{ha}^{-1}, \mathrm{MAP}^{2} \mathrm{ZnSO}_{4}$ at $2.2 \mathrm{~kg} \mathrm{Zn} \mathrm{ha}^{-1}, \mathrm{DAP}+\mathrm{ZnSO}_{4}$ at $2.2 \mathrm{~kg} \mathrm{Zn}$ $\mathrm{ha}^{-1}$, DAP, and N-only. Treatments that included AMS, MES10, and MESZ had similar soil test S concentrations. Post-harvest soil samples showed no significant differences among treatments for potassium $(P=0.7)$, magnesium $(P=0.75)$, calcium $(P=0.54)$, or organic matter $(P=0.2)$ levels (data not presented).

\subsection{Soybean Response Following Corn}

Soybean plant population ranged from 303,000 to 340,200 plants $\mathrm{ha}^{-1}$, but was non-significant $(P=0.52)$ between treatments (Table 9). At Novelty and Albany in 2014 and 2015, whole-plant samples taken before physiological maturity at R6 (Fehr \& Caviness, 1971) were analyzed for P, S, and Zn concentration. At Novelty in 2014 and 2015, no differences were seen in plant $\mathrm{P}(P=0.69)$ or $\mathrm{S}(P=0.26)$ concentration (Table 10). At Albany in 2015, no significance differences appeared between plant $\mathrm{P}(P=0.64), \mathrm{S}(P=0.66)$, or $\mathrm{Zn}(P=0.98)$ concentration; however, at Albany in 2014, P, S, and Zn concentrations were significantly different. At Albany in 2014, plant $\mathrm{P}$ concentration ranged from 2.48 to $3.81 \mathrm{~g} \mathrm{~kg}^{-1}$. Plant $\mathrm{P}$ concentration in the DAP+AMS treatment was 0.57 to $1.33 \mathrm{~g} \mathrm{~kg}^{-1}$ greater than all treatments. The $\mathrm{N}$-only control had a plant $\mathrm{Zn}$ concentration similar to 7 of the 16 treatments, indicating there may be no strong effect regarding application of $\mathrm{P}$ for soybeans between treatments applied for corn and plant P concentrations observed in soybean the following year. A 14-site study in Iowa showed increased P uptake at eight sites (Borges \& Mallarino, 2003).

At Albany in 2014, whole-plant $S$ concentration ranged from 1.95 to $2.99 \mathrm{~g} \mathrm{~kg}^{-1}$ (Table 10). Similar to plant $\mathrm{P}$ concentration, DAP+AMS had the greatest plant $\mathrm{S}$ concentration, which was 0.33 to $1 \mathrm{~g} \mathrm{~kg}^{-1}$ greater than all treatments except MAP+SuperZn at $5.6 \mathrm{~kg} \mathrm{Zn} \mathrm{ha}^{-1}$ and DAP+SuperZn at $5.6 \mathrm{~kg} \mathrm{Zn} \mathrm{ha}^{-1}$. Interestingly, treatments including SuperZn generally had a greater plant $\mathrm{S}$ concentration than treatments including $\mathrm{ZnSO}_{4}$. Again, this 
could point to the lack of a good relationship between fertilizer treatments and plant S concentrations. Similarly, when S fertilizer was applied to wheat in a wheat-soybean rotation, no significant differences were reported in soybean S concentrations (Singh et al., 2014).

Plant $\mathrm{Zn}$ concentration was significantly affected at both locations in 2014 and Novelty in 2015 (Table 10). At Albany in 2014, plant Zn concentrations ranged from 24.2 to $35.6 \mathrm{mg} \mathrm{kg}^{-1}$. Similar to both plant P and S concentration data, DAP+AMS had the highest plant $\mathrm{Zn}$ concentration at $35.6 \mathrm{mg} \mathrm{kg}^{-1}$, which was 5.0 to $11.4 \mathrm{mg}$ $\mathrm{kg}^{-1}$ greater than all treatments except DAP+SuperZn at $5.6 \mathrm{~kg} \mathrm{Zn} \mathrm{ha}^{-1}$ and MAP+SuperZn at 2.2 or $5.6 \mathrm{~kg} \mathrm{Zn}$ $\mathrm{ha}^{-1}$. In a planned contrast comparing $\mathrm{Zn}$ rate $\left(2.2\right.$ vs. $\left.5.6 \mathrm{~kg} \mathrm{Zn} \mathrm{ha}^{-1}\right)$ and $\mathrm{Zn}$ source (SuperZn vs. $\left.\mathrm{ZnSO}_{4}\right)$, SuperZn had significantly higher plant $\mathrm{Zn}$ concentration than $\mathrm{ZnSO}_{4}(P=0.0005)$, while $\mathrm{Zn}$ rate showed no significant difference $(P=0.12)$. At Novelty in 2014 and 2015 , plant $\mathrm{Zn}$ concentration ranged from 26.1 to 34.7 $\mathrm{mg} \mathrm{kg}{ }^{-1}$. MAP+SuperZn at $5.6 \mathrm{~kg} \mathrm{Zn} \mathrm{ha}^{-1}$ increased plant $\mathrm{Zn}$ concentration by 4.9 to $10.3 \mathrm{mg} \mathrm{kg}^{-1}$ over all treatments except $\mathrm{DAP}+\mathrm{ZnSO}_{4}$ at $5.6 \mathrm{~kg} \mathrm{Zn} \mathrm{ha}^{-1}$. In a planned contrast comparing $\mathrm{Zn}$ rate (2.2 vs $\left.5.6 \mathrm{~kg} \mathrm{Zn} \mathrm{ha}^{-1}\right)$ and $\mathrm{Zn}$ source (SuperZn vs $\mathrm{ZnSO}_{4}$ ), $\mathrm{Zn}$ at $5.6 \mathrm{~kg} \mathrm{Zn} \mathrm{ha}^{-1}$ increased plant $\mathrm{Zn}$ concentration compared to $2.2 \mathrm{~kg} \mathrm{Zn}$ $\mathrm{ha}^{-1}(P=0.0008)$, while $\mathrm{Zn}$ source was not significant $(P=0.72)$. These results showed completely different responses at Novelty compared to Albany.

Table 10. Soybean whole plant $\mathrm{P}, \mathrm{S}$, and $\mathrm{Zn}$ concentrations as affected by $\mathrm{Zn}$ treatments at Albany and Novelty in 2014-2015

\begin{tabular}{|c|c|c|c|c|c|c|c|c|c|c|}
\hline \multirow{3}{*}{$\mathrm{Zn}$ treatments ${ }^{\dagger}$} & \multirow{3}{*}{ Zn amount } & \multicolumn{3}{|c|}{ Leaf $P$} & \multicolumn{3}{|c|}{ Leaf S } & \multicolumn{3}{|c|}{ Leaf $\mathrm{Zn}$} \\
\hline & & \multirow{2}{*}{ Novelty } & \multicolumn{2}{|c|}{ Albany } & \multirow{2}{*}{ Novelty } & \multicolumn{2}{|c|}{ Albany } & \multirow{2}{*}{ Novelty } & \multicolumn{2}{|c|}{ Albany } \\
\hline & & & 2014 & 2015 & & 2014 & 2015 & & 2014 & 2015 \\
\hline & $\mathrm{kg} \mathrm{ha}^{-1}$ & |------- & $\mathrm{kg}^{-1}$ & ב---- & - --------- & $\mathrm{kg}^{-1}-$ & ב---- & |-------- & $\mathrm{ng} \mathrm{kg}^{-1}$ & ב-"--- \\
\hline Non-treated control & 0 & 3.1 & 2.58 & 3.8 & 2.2 & 2.10 & 2.5 & 27.3 & 29.5 & 24.9 \\
\hline N-only & 0 & 3.1 & 2.83 & 3.6 & 2.2 & 2.14 & 2.5 & 28.6 & 28.8 & 24.7 \\
\hline DAP & 0 & 3.1 & 2.55 & 3.7 & 2.1 & 2.18 & 2.5 & 26.1 & 26.0 & 25.4 \\
\hline $\mathrm{DAP}+\mathrm{ZnSO}_{4}$ & 2.2 & 3.3 & 2.98 & 3.6 & 2.4 & 2.32 & 2.5 & 34.7 & 28.3 & 24.8 \\
\hline $\mathrm{DAP}+\mathrm{ZnSO}_{4}$ & 5.6 & 3.1 & 2.65 & 3.8 & 2.3 & 2.28 & 2.5 & 29.6 & 27.7 & 25.4 \\
\hline MAP & 0 & 3.0 & 2.48 & 3.7 & 2.1 & 1.94 & 2.4 & 29.1 & 24.2 & 24.9 \\
\hline $\mathrm{MAP}+\mathrm{ZnSO}_{4}$ & 2.2 & 3.2 & 2.60 & 3.4 & 2.3 & 2.14 & 2.4 & 31.5 & 25.7 & 26.1 \\
\hline $\mathrm{MAP}+\mathrm{ZnSO}_{4}$ & 5.6 & 3.2 & 2.48 & 3.6 & 2.2 & 2.28 & 2.5 & 28.9 & 27.0 & 25.0 \\
\hline MESZ & 2.2 & 3.2 & 2.73 & 3.7 & 2.3 & 2.08 & 2.5 & 28.2 & 25.0 & 26.1 \\
\hline MES10 & 0 & 3.2 & 3.14 & 3.7 & 2.2 & 2.35 & 2.5 & 28.5 & 29.0 & 25.0 \\
\hline $\mathrm{MAP}+\mathrm{AMS}$ & 0 & 3.2 & 3.24 & 3.6 & 2.3 & 2.63 & 2.5 & 27.5 & 30.6 & 26.1 \\
\hline MAP + SuperZn & 2.2 & 3.0 & 3.0 & 3.7 & 2.2 & 2.40 & 2.5 & 26.3 & 31.3 & 25.4 \\
\hline MAP + SuperZn & 5.6 & 3.3 & 3.59 & 3.6 & 2.3 & 2.80 & 2.5 & 36.4 & 32.0 & 24.4 \\
\hline $\mathrm{DAP}+\mathrm{AMS}$ & 0 & 3.3 & 3.81 & 3.9 & 2.3 & 2.99 & 2.5 & 26.5 & 35.6 & 25.0 \\
\hline $\mathrm{DAP}+$ SuperZn & 2.2 & 3.2 & 2.86 & 3.6 & 2.2 & 2.67 & 2.5 & 30.5 & 29.0 & 25.4 \\
\hline $\mathrm{DAP}+$ SuperZn & 5.6 & 3.2 & 3.03 & 3.5 & 2.2 & 2.67 & 2.4 & 30.7 & 34.5 & 24.6 \\
\hline $\operatorname{LSD}(P=0.05)$ & & NS & 0.04 & NS & NS & 0.03 & NS & 3.4 & 4.7 & NS \\
\hline
\end{tabular}

Note. ${ }^{\dagger}$ Abbreviations: AMS; Ammonium sulfate; DAP, diammonium phosphate; MAP, monoammonium phosphate.

* Data were combined over years.

Nutrient concentration and total plant tissue weights were used to calculate total plant uptake of $\mathrm{P}, \mathrm{S}$, and $\mathrm{Zn}$ (Table 11). Although significant differences were observed in plant nutrient concentrations, all treatments were similar when total plant uptake was calculated [P $(P=0.52), \mathrm{S}(P=0.49)$, and $\mathrm{Zn}(P=0.60)]$. In 2012 and 2013, grain yields ranged from 2,380 to $2,490 \mathrm{~kg} \mathrm{ha}^{-1}$, and all treatments yielded similarly $(P=0.85)$ (Table 9). Seed oil and protein content were measured to determine any impact of treatments on seed quality. Seed oil ranged from 191 to $197 \mathrm{~g} \mathrm{~kg}^{-1}$, with no difference between treatments $(P=0.42)$. Protein concentration ranged from 352 to $360 \mathrm{~g} \mathrm{~kg}^{-1}$, but also showed no effects of the treatments $(P=0.46)$. In 2014 and 2015, soybean population, yield, oil, and protein combined over years and locations (Novelty and Albany). Soybean plant population 
ranged from 293,300 to 336,800 plants $\mathrm{ha}^{-1}$ and showed no difference among treatments $(P=0.15)$. Similarly, yields $\left(2,900\right.$ to $\left.3,200 \mathrm{~kg} \mathrm{ha}^{-1}\right)$ showed no differences among treatments $(P=0.16)$ (Table 9). Grain quality (oil, protein) was also similar among treatments $(P=0.37, P=0.46$, respectively). Between treatments, seed oil concentration had a small range, 180 to $190 \mathrm{~g} \mathrm{~kg}^{-1}$, and protein concentration had a narrower range, $346 \mathrm{go} 349 \mathrm{~g}$ $\mathrm{kg}^{-1}$.

Fertilizer applications to corn in a corn-soybean rotation had inconsistent effects on soybean yield and seed quality. Anthony et al. (2012) and Buah, Polito, and Killorn (2000) reported no difference in yield between 0 and $56 \mathrm{~kg} \mathrm{P} \mathrm{ha}{ }^{-1}$. However, others have shown increased yields, but at low soil test P levels (Borges \& Mallarino, 2000, 2003). An Iowa study at over 112 locations showed positive yield effects from P fertilizer at only 20 sites (Haq \& Mallarino, 2005). Applications of S fertilizer have also had mixed results. Divito, Echeverria, Andrade, and Sadras (2015) reported increased yields with S application, while Singh et al. (2014) reported no response to $\mathrm{S}$ fertilizer.

Table 11. Total soybean plant P, S, and Zn uptake at Novelty and Albany in 2014-2015. Data were combined over all site-years

\begin{tabular}{|c|c|c|c|c|}
\hline $\mathrm{Zn}$ treatments ${ }^{\dagger}$ & Zn amount & P uptake & S uptake & Zn uptake \\
\hline & $\mathrm{kg} \mathrm{ha}^{-1}$ & \multicolumn{3}{|c|}{ - } \\
\hline Non-treated control & 0 & 17.5 & 12.7 & 1.2 \\
\hline N-only & 0 & 18.1 & 13.4 & 1.3 \\
\hline DAP & 0 & 21.8 & 15.4 & 1.5 \\
\hline $\mathrm{DAP}+\mathrm{ZnSO}_{4}$ & 2.2 & 19.0 & 13.9 & 1.5 \\
\hline $\mathrm{DAP}+\mathrm{ZnSO}_{4}$ & 5.6 & 19.8 & 14.5 & 1.4 \\
\hline MAP & 0 & 18.0 & 12.9 & 1.4 \\
\hline $\mathrm{MAP}+\mathrm{ZnSO}_{4}$ & 2.2 & 18.7 & 13.8 & 1.3 \\
\hline $\mathrm{MAP}+\mathrm{ZnSO}_{4}$ & 5.6 & 21.1 & 15.0 & 1.5 \\
\hline MESZ & 2.2 & 19.8 & 14.8 & 1.4 \\
\hline MES10 & 0 & 20.5 & 14.8 & 1.4 \\
\hline MAP + AMS & 0 & 20.0 & 14.3 & 1.4 \\
\hline MAP + SuperZn & 2.2 & 20.8 & 15.3 & 1.4 \\
\hline MAP + SuperZn & 5.6 & 19.2 & 14.2 & 1.6 \\
\hline $\mathrm{DAP}+\mathrm{AMS}$ & 0 & 19.0 & 13.8 & 1.2 \\
\hline DAP + SuperZn & 2.2 & 21.8 & 15.8 & 1.6 \\
\hline DAP + SuperZn & 5.6 & 21.7 & 15.5 & 1.6 \\
\hline$P$-value & & 0.52 & 0.49 & 0.60 \\
\hline
\end{tabular}

Note. ${ }^{\dagger}$ Abbreviations: AMS; Ammonium sulfate; DAP, diammonium phosphate; MAP, monoammonium phosphate.

\section{Conclusion}

Variation in precipitation over site-years strongly affected corn grain yields. Ear leaf P tissue concentration at VT showed fertilized treatments within the sufficiency range, with no significant difference among treatments. Ear leaf S tissue concentration at VT showed no significant differences, except for the non-treated control. All fertilized treatments were within the ear leaf S sufficiency range. All treatments were similar for ear leaf $\mathrm{Zn}$ concentration at VT, except for the non-treated control, which was significantly lower than all other treatments and for MES10, which was significantly lower than $\mathrm{N}$-only and $\mathrm{DAP}+\mathrm{ZnSO}_{4}$ at $5.6 \mathrm{~kg} \mathrm{Zn} \mathrm{ha}^{-1}$. Yields showed no significant differences at Novelty in 2013, and all treatments were similar at Novelty in 2011. In 2012, because of a severe drought, yields in the non-treated control were the greatest. At Novelty in 2014 and Albany in 2013 and 2014, adding S and/or Zn had no effect on yield. The rate of $\mathrm{Zn}$ fertilizer (2.2 vs $5.6 \mathrm{~kg} \mathrm{Zn} \mathrm{ha}^{-1}$ ) also showed no significant effect on yield. When $\mathrm{P}$ was determined post-harvest, soil samples reflected the application of $\mathrm{P}$ fertilizer. Generally, when Zn was applied, soil test Zn increased. At Novelty, MES10 and MESZ had the greatest increase in soil test $\mathrm{S}$, while at Albany MAP+AMS had the greatest amount of soil test S. Carry-over fertilizer from corn experiments showed differences in plant nutrient concentrations, but this had no 
effect on total plant nutrient (P, S, or Zn) uptake, grain yield or quality. However, at Novelty plant $\mathrm{Zn}$ concentration was increased with $5.6 \mathrm{~kg} \mathrm{Zn} \mathrm{ha}^{-1}$ compared to $2.2 \mathrm{~kg} \mathrm{Zn} \mathrm{ha}{ }^{-1}$, while Albany showed an increase in soybean $\mathrm{Zn}$ concentration with SuperZn when compared to $\mathrm{ZnSO}_{4}$. This indicates that micro-nutrient uptake was affected by $\mathrm{Zn}$ rate and source, depending on the soil type.

\section{References}

Amijee, F., Stribley, D. P., \& Tinker, P. B. (1990). Soluble carbohydrates in roots of leek (Allium porrum) plants in relation to phosphorus supply and VA mycorrhizas. Plant Nutrition-Physiology and Applications. New York, NY: Springer.

Anthony, P., Maizer, G., Sparrow, S., \& Zhang, M. (2012). Soybean yield and quality in relation to soil properties. Agronomy Journal, 104, 1443-1458. http://dx.doi.org/10.1007/978-94-009-0585-6_27

Borges, R., \& Mallarino, A. P. (2000). Grain yield, early growth, and nutrient uptake of no-till soybean as affected by phosphorus and potassium placement. Agronomy Journal, 92, 380-388. http://dx.doi.org/10.2134/agronj2000.922380x

Borges, R., \& Mallarino, A. P. (2003). Broadcast and deep-band placement of phosphorus and potassium for soybean managed with ridge tillage. Soil Science Society America Journal, 67, 1920-1927. http://dx.doi.org/10.2136/sssaj2003.1920

Briskin, D. P., Bloom, A., Taiz, L., \& Zeiger, E. (2010). Mineral Nutrition. Plant Physiology (5th ed.). Sunderland, MA: Sinauer Associates Inc.

Broadley, M. R., White, P. J., Hammond, J. P., Zelko, I., \& Lux, A. (2007). Zinc in plants. New Phytologist, 173, 677-702. http://dx.doi.org/10.1111/j.1469-8137.2007.01996.x

Bryson, G. M., Mills, H. A., Sasseville, D. N., Jones, J. B., \& Barker, A. V. (2014). Plant Analysis Handbook III: A Guide to Sampling, Preparation, Analysis, Interpretation and Use of Results of Agronomic and Horticultural Crop Plant Tissue. Athens, GA: Micro-Macro Publishing, Inc.

Buah, S. S., Polito, T. A., \& Killorn, R. (2000). No-tillage soybean response to banded and broadcast and direct and residual fertilizer phosphorus and potassium applications. Agronomy Journal, 92, 657-662. http://dx.doi.org/10.2134/agronj2000.924657x

Buchholz, D. D., Brown, J. R., Garret, J., Hanson, R., \& Wheaton, H. (2004). Soil Test Interpretations and Recommendations Handbook. Columbia, MO: University of Missouri-College of Agriculture, Division of Plant Sciences.

Camberato, J., \& Casteel, S. (2010). Keep an eye open of sulfur deficiency in wheat. Soil Fertility Update. Purdue University Extension. Retrieved from https://www.agry.purdue.edu/ext/soybeanArrivals/04-13-10 JC_SC_Sulfur_deficiency.pdf

Camberato, J., Maloney S., Casteel, S., \& Johnson, K. (2012). Soil Fertility Update. Purdue University Extension. Retrieved from https://www.agry.purdue.edu/ext/soilfertility/05-03-12Sulfur_deficiency_alfa lfa.pdf

Divito, G. A., Echeverría, H. E., Andrade, F. A., \& Sadras, V. O. (2015). Diagnosis of S deficiency in soybean crops: Performance of S and N: S determinations in leaf, shoot and seed. Field Crops Research, 180, 167-175. http://dx.doi.org/10.1016/j.fcr.2015.06.006

Fageria, N. K. (2004). Dry matter yield and nutrient uptake by lowland rice at different growth stages. Journal of Plant Nutrition, 27, 947-958. http://dx.doi.org/10.1081/PLN-120037529

Fehr, W. R., \& Caviness, C. E. (1971). Stages of soybean development. Crop Sci., 11, 929-930. http://dx.doi.org/ 10.2135/cropsci1971.0011183X001100060051x

Freeman, K., Ruffo, M., \& Mann, K. (2014). Improving crop yield and nutrient uptake efficiency with premium sulfur enhanced phosphate fertilizer. American Society of Agronomy. Abstract, Long Beach, CA.

Graham, R. D., Ascher, J. S., \& Hynes, S. C. (1992). Selecting zinc-efficient cereal genotypes for soils of low zinc status. Plant and Soil, 146, 241-250. http://dx.doi.org/10.1007/BF00012018

Halim, A. H., Wassom, C. E., \& Ellis Jr., R. (1968). Zinc deficiency symptoms and zinc and phosphorous interactions in several strains of corn (Zea mays L.). Agronomy Journal, 60, 267-271. http://dx.doi.org/ 10.2134/agronj1968.00021962006000030007x 
Haq, M. U., \& Mallarino, A. P. (2005). Response of soybean grain oil and protein concentrations to foliar and soil fertilization. Agronomy Journal, 97, 910-918. http://dx.doi.org/10.2134/agronj2004.0215

Hergert, G. W. (2000). Fertility principals: Sulfur. Nutrient Management of Agronomic Crops in Nebraska. University of Nebraska Extension. Retrieved from http://extensionpublications.unl.edu/assets/ pdf/ec155.pdf

Huang, C., Barker, S. J., Langridge, P., Smith, F. W., \& Graham, R. D. (2000). Zinc deficiency up-regulates expression of high-affinity phosphate transporter genes in both phosphate-sufficient and-deficient barley roots. Plant Physiology, 124, 415-422. http://dx.doi.org/10.1104/pp.124.1.415

Jones Jr., J. B. (1967). Interpretation of plant analysis for several agronomic crops. Soil Testing and Plant Analysis. Part II. SSSA Special Publ. Series No. 2. Madison, WI: Soil Sci. Soc., Amer.

Kaiser, D. S., Strock, J., \& Lamb, J. (2008). Impact of phosphorus fertilization strategies on efficiency of nitrogen use by corn rotated with soybeans. Minnesota Department of Agriculture. Retrieved from http://www.mda.state.mn.us/chemicals/fertilizers/afrec/researchprojects/ /media/Files/chemicals/afrec/repor ts/phosfertstratimpact.ashx

Keefer, R. F., Singh, R. N., Horvath, D. J., \& Henderlong, P. R. (1972). Response of corn to time and rate of phosphorous and zinc application. Soil Science Society of America Journal, 36, 628-632. http://dx.doi.org/10.2136/sssaj1972.03615995003600040036x

Mahler, R. L. (2004). Nutrients plants require for growth. University of Idaho Extension. Retrieved from http://www.cals.uidaho.edu/edComm/pdf/CIS/CIS1124.pdf

McBeath, T. M., \& McLaughlin, M. J. (2014). Efficacy of zinc oxides as fertilizers. Plant and Soil, 374, 843-855. http://dx.doi.org/10.1007/s11104-013-1919-2

Mosaic. (2007). A New Vision of Phosphate from Mosaic. Plymouth, MN: The Mosaic Company.

Nathan, M. V., Stecker, J. A., \& Sun, Y. (2012). Soil Testing in Missouri. Univ. Extension, Division of Plant Sciences, College of Agriculture Food and Natural Resources, University of Missouri, Columbia, MO.

Olsen, S. (1982). Micronutrient interaction. In J. M. Mortved, \& W. L. Lindsay (Eds.), Micronutrients in agriculture (pp. 243-264). Soil Science Society America, Madison, WI.

Pierzynski, G. M., McDowell, R. W., Sims, J. T., \& Sharpley, A. N. (2005). Chemistry, cycling, and potential movement of inorganic phosphorus in soils. Phosphorus: Agriculture and the Environment (pp. 53-86). ASA, Madison, WI.

Rehm, G. W., Sorensen, R. C., \& Wiese, R. A. (1981). Application of phosphorous, potassium and zinc to corn grown for grain or silage: early growth and yield. Soil Sci. Soc. Am. J., 45, 523-528. http://dx.doi.org/10.2136/sssaj1981.03615995004500030017x

Rehm, G. W., Sorensen, R. C., \& Wiese, R. A. (1983). Application of phosphorous, potassium and zinc to corn grown for grain or silage: Nutrient concentration and uptake. Soil Sci. Soc. Am. J., 47, 697-700. http://dx.doi.org/10.2136/sssaj1983.03615995004700040019x

Robson, A. D., \& Pitman, M. G. (1983). Interactions between nutrients in higher plants. Inorganic plant nutrition (pp. 147-180). Springer. http://dx.doi.org/10.1007/978-3-642-68885-0_6

Sawyer, J., \& Barker, D. (2009). Evaluation of Mosaic MicroEssentials sulfur fertilizer products for corn production (p. 5). Iowa State University Department of Agronomy, Ames, IA.

Sawyer, J., \& Barker, D. (2010). Evaluation of combination phosphorus-sulfur fertilizer products for corn production (p. 4). Iowa State University Department of Agronomy, Ames, IA.

Sawyer, J., \& Barker, D. (2012). Sulfur fertilization response in Iowa corn and soybean production. Proceedings of the 2012 Wisconsin Crop Management Conference (pp. 39-48). Madison, WI: University of Wisconsin-Madison.

Schachtman, D. P., Reid, R. J., \& Ayling, S. M. (1998). Phosphorus uptake by plants: From soil to cell. Plant Physiology, 116, 447-453. http://dx.doi.org/10.1104/pp.116.2.447

Schnappinger, M. G., Martens, D. C., \& Hawkins, G. W. (1969). Response of corn to Zn-EDTA and ZnSO in $^{2}$ field investigations. Agron. J., 61, 834-836. http://dx.doi.org/10.2134/agronj1969.00021962006100060002x

Schulte, E. E. (2004). Soil and applied zinc (p. 2). University of Wisconsin Extension. 
Schulte, E. E., \& Kelling, K. A. (1992). Understanding plant nutrients: Soil and applied phosphorus (No. A2520, p. 32). Univ. Wis. Extn. Pub.

Singh, J. P., Karamanos, R. E., \& Stewart, J. W. B. (1986). Phosphorus-induced zinc deficiency in wheat on residual phosphorus plots. Agronomy Journal, 78, 668-675. http://dx.doi.org/10.2134/agronj1986.0002196 $2007800040023 x$

Singh, J. P., Karamanos, R. E., \& Stewart, J. W. B. (1988). The mechanism of phosphorus-induced zinc deficiency in bean (Phaseolus vulgaris L.). Canadian Journal of Soil Science, 68, 345-358. http://dx.doi.org/10.4141/cjss88-032

Singh, S. P., Singh, R., Singh, M. P., \& Singh, V. P. (2014). Impact of sulfur fertilizer on different forms and balance of soil sulfur and the nutrition of wheat in wheat-soybean cropping sequence in Tarai soil. Journal of Plant Nutrition, 37, 618-632. http://dx.doi.org/10.1080/01904167.2013.867987

Slaton, N. A., Norman, R. J., Roberts, T. L., DeLong, R. E., Massey, C., Clark, S., \& Branson, J. (2010). Evaluation of new fertilizers and different methods of application for rice production. B.R. Wells Rice Research Studies (pp. 266-277).

Stout, W. L., \& Bennett, O. L. (1983). Effect of Mg and Zn fertilization on soil test levels, ear leaf composition, and yields of corn in northern West Virginia. Communications in Soil Science \& Plant Analysis, 14, 601-613. http://dx.doi.org/10.1080/00103628309367392

Tabatabai, M. A. (1984). Importance of Sulphur in Crop Production. Biogeochemistry, 1, 45-62. http://dx.doi.org/10.1007/BF02181120

Uribelarrea, M., Below, F. E., \& Moose, S. P. (2004). Grain composition and productivity of maize hybrids derived from the Illinois protein strains in response to variable nitrogen supply. Crop Sci., 44, 1593-1600. http://dx.doi.org/10.2135/cropsci2004.1593

USDM. (2015). United States Drought Monitor. Retrieved from http://doughtmonitor.unl.edu

Vance, C. P., Uhde-Stone, C., \& Allan, D. L. (2003). Phosphorus acquisition and use: critical adaptations by plants for securing a nonrenewable resource. New Phytologist, 157, 423-447. http://dx.doi.org/10.1046/ j.1469-8137.2003.00695.x

Woolfork, C., Olson, R., Mann, K., \& Perez, O. (2014). Canola (Brassica napus) fertilizer seed safety. ASA, CSSA, SSSA. Abstract. Long Beach, CA.

Yerokun, O. A., \& Christenson, D. R. (1990). Relating High Soil Test Phosphorus Concentrations to Plant Phosphorus Uptake. SSSAJ, 54, 796-799. http://dx.doi.org/10.2136/sssaj1990.03615995005400030029x

\section{Copyrights}

Copyright for this article is retained by the author(s), with first publication rights granted to the journal.

This is an open-access article distributed under the terms and conditions of the Creative Commons Attribution license (http://creativecommons.org/licenses/by/4.0/). 